\title{
Heat Flow Constraints on the South Pacific Superswell
}

\author{
CAROL A. STEIN \\ Department of Geological Sciences, University of Illinois at Chicago \\ DALLAS H. ABBOTT \\ Lamont-Doherty Geological Observatory, Palisades, New York
}

\begin{abstract}
The South Pacific superswell has been defined as a large region of anomalously shallow bathymetry, low Love wave velocities, and low effective elastic thicknesses relative to those predicted for its age. These phenomena have been interpreted as reflecting a combination of lithospheric reheating and thinning, and dynamic uplift due to mande flow. We use heat flow data to better constrain the thermal structure of this region and examine the predictions of various possible models. The average heat flow for the superswell region does not differ significanly from that for lithosphere of similar ages elsewhere on the Pacific plate. Given their uncertainties, the heat fow data imply that thermal lithospheric thickness exceeds $60 \mathrm{~km}$, but cannot discriminate between greater thicknesses. The lack of observed high heat flow appears not to be explained by biases due to water circulation in the thin sediment cover, since the superswell heat flow is not higher than for sites elsewhere with similar sedimentary environments. The Darwin Rise has been proposed as a fossil superswell in the Cretaceous, on the basis of the many similar characteristics to the South Pacific superswell. We find that the Darwin Rise heat flow values do not exceed those for similar ages elsewhere in the Pacific and Atlantic. This observation suggests that any thermal effects associated with the formation of the Darwin Rise are no longer present, and it is consistent with the idea of a fossil superswell. The surface heat flow data thus provide no evidence that the temperatures in the uppermost portion of the lithosphere are significantly higher in the entire superswell region than in other areas of comparable age. This observation is intriguing given the suggestion that the thin effective elastic thicknesses inferred from seamount loading may reflect reheating of the lithosphere. Models in which the plate thicknesses and/or the basal temperatures are increased to yield temperatures high enough to explain the low effective elastic thicknesses predict surface heat flow much higher than observed. Reheating the lithosphere, as is proposed for hot spots, yields temperatures adequate to explain the effective elastic thicknesses only if reheating occurs at very shallow depths, and again implies a surface heat flow much greater than observed. Hence, unless shallow reheating is somehow localized beneath the seamounts, the thinner elastic thicknesses may reflect mechanical, rather than thermal, weakening of the lithosphere.
\end{abstract}

\section{INTRODUCTION}

Heat flow and bathymetric data provide constraints for thermal models of the formation and cooling of oceanic lithosphere and hence on its thermal structure [e.g., Sclater and Francheteau, 1970; Parsons and Sclater, 1977]. The depth of most oceanic seafloor is well fit by such models. However, a large region of the Pacific Ocean (west of the East Pacific Rise to $\sim 160^{\circ} \mathrm{W}, \sim 9^{\circ}-\sim 30^{\circ} \mathrm{S}$ ) is substantially shallower than might be expected for its age, which ranges from 20 to $90 \mathrm{Ma}$. This area (Figure 1), termed the South Pacific superswell by McNutt and Fisher [1987], contains the Cook-Austral, Marquesas, Pitcaim, and Society seamount chains (Figure 2), which have been interpreted as hot spot tracks with volcanic edifices formed in the last 18 m.y. [Morgan, 1972; Duncan and Clague, 1985; Gordon and Henderson, unpublished manuscript, 1985]. Holocene volcanism occurs at several sites on the chains, such as Mehetia and MacDonald seamount. The NW-SE trend of the plateaus and island chains is at a high angle to both the isochrons and fracture zones, and is similar to that of the Hawaiian hot spot track.

The effective elastic thicknesses of the lithosphere calculated from the loading of these volcanoes and seamounts are substantially less than can be expected for the age of loading [McNutt and Menard, 1978; Calmant and Cazenave, 1987; Calmant et

Copyright 1991 by the American Geophysical Union.

Paper number 91JB00774. 0148-0227/91/91JB-00774\$05.00 al., 1990]. In addition, this area is associated with the Dupal geochemical anomaly [Hart, 1984, 1988], a geoid low [McNutt and Judge, 1990], a low-velocity zone for Love waves [Nishimura and Forsyth, 1985], and a correlation between the maximum Dupal anomaly location and low seismic velocities in the lower mantle [Castillo, 1988].

McNutt and Fisher [1987] inferred, from an analysis of bathymetric depth anomalies, that the superswell region was bounded by the Marquesas fracture zone to the north. The largest depth and negative geoid anomalies are between the Marquesas and the Austral fracture zones, but the superswell probably continues south of the Austral fracture zone (M. McNutt, personal communication, 1989). McNutt and Fisher proposed that the shallow bathymetry can be modeled if the lithosphere in the area has on average the temperature structure of a $75-\mathrm{km}$ plate with a $1385^{\circ} \mathrm{C}$ basal temperature, in contrast to the standard oceanic thermal model of a $125-\mathrm{km}$-thick plate with basal temperature of $\sim 1350^{\circ} \mathrm{C}$. McNutt and Judge [1990] further suggest that the surface wave velocities and flexural strength data can be explained by a thermal plate $75 \mathrm{~km}$ thick and that the observed geoid low arises from a deeper low-density "superplume." In their model the thinned lithosphere is a consequence of enhanced heat flux from the convecting mantle and low viscosity beneath the plate, both of which prevent the growth of a stable lithospheric thermal boundary layer to thicknesses greater than $75 \mathrm{~km}$. They suggest that small-scale convective instabilities in the low-viscosity zone may be an important mechanism responsible for maintaining a thinner plate. Such a mechanism was proposed for the origin of lineations, observed in altimetry data over a large region west of the East Pacific Rise including 
the superswell, with a trend approximately parallel to the absolute plate motion [Haxby and Weissel, 1986]. McAdoo and Sandwell [1989] suggest that although the lineations may be produced by small-scale convection very near the East Pacific

\section{HEAT FLOW LOCATIONS}

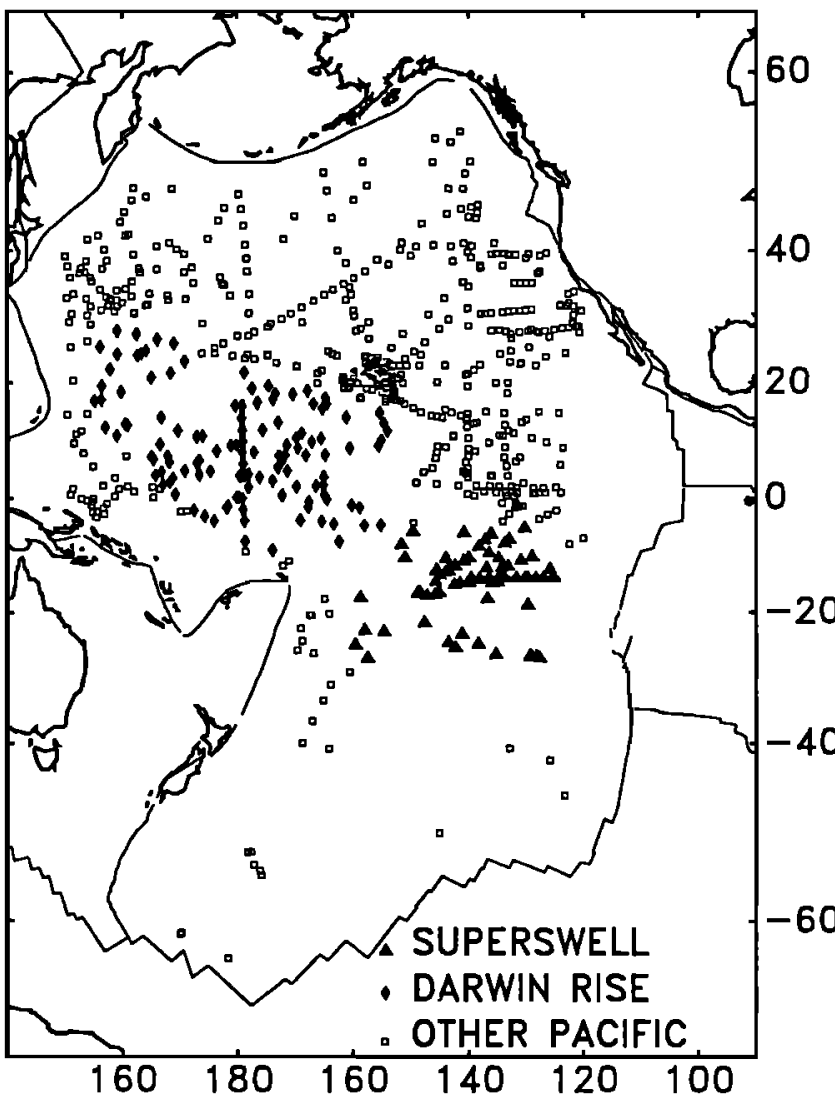

Fig. 1. Location of heat flow measurements used in this study on the Pacific plate, showing the superswell and Darwin Rise regions.

\section{SUPERSWELL REGION}

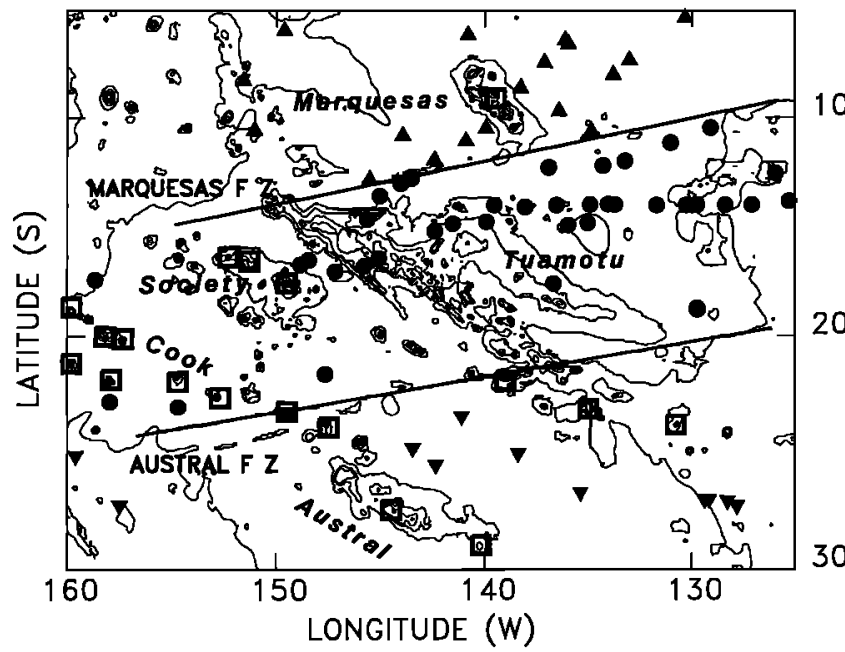

Fig. 2. South Pacific superswell bathymetry and heat flow sites. Heat flow sites north of the Marquesas fracture zone are represented by solid upward-pointing triangles, south of the Austral fracture zone by solid downward-pointing triangles, and between the two fracture zones by solid circles. Open squares indicate the locations of effective elastic thickness measurements [Calmant and Cazenave, 1986; Calmant, 1987]. Contour interval is $1000 \mathrm{~m}$.
Rise, they prefer an origin on older, anomalously weak lithosphere perhaps resulting primarily from shallow lithospheric loads, in the crust or near the Moho. One possible load might result from tension in the north-south direction [Dunbar and Sandwell, 1988].

McNutt and Fisher [1987] also suggested that the Darwin Rise [Menard, 1964, 1984], a large region of rough relief in the westem Pacific (Figure 1) including the Mid-Pacific Mountains, the Marshall Islands, the Magellan Seamounts, and the Wake Guyots, has similar characteristics to the present superswell and is a fossil superswell from the Cretaceous. Many of the hot spot tracks within the present-day South Pacific superswell can be traced back to the Darwin Rise [Duncan and Clague, 1985; Gordon and Henderson, unpublished manuscript, 1985]. The depths for the Darwin Rise are shallower than can be expected for its age [Renkin and Sclater, 1988], and the petrologic anomalies are similar to those in the superswell [e.g., Hart, 1984; Natland and Wright, 1984; Staudigel et al., 1991]. The effective elastic thicknesses for this region are lower than can be expected for the age of loading [Smith et al., 1989; Wolfe and McNutt, 1991]. McNutt et al. [1990] suggested that the Darwin Rise was dynamically uplifted about $113 \pm 8 \mathrm{Ma}$ and "superswell conditions" were present until about $70-80 \mathrm{Ma}$, implying that a superswell is a transient feature. The lack of velocity anomalies for Love waves beneath the Darwin Rise, unlike the present South Pacific superswell [Nishimura and Forsyth, 1985], also suggests that there is no present thermal anomaly in this region [McNutt et al., 1990].

In these models, a superswell incorporates several aspects previously proposed in discussions of the origin of intraplate hot spots and swells. Thus, a superwell may be considered as a larger version of hot spots and swells. Models for the origin of such features generally rely on two primary effects, reheating mechanisms which elevate temperatures in the lower part of the lithosphere to asthenospheric temperatures [Crough, 1978; Detrick and Crough, 1978] or dynamic support due to mantle flow [Parsons and Daly, 1983; Courtney and White, 1986; Robinson and Parsons, 1988; Liu and Chase, 1989]. Heat flow data have been used to try and distinguish between these two end-member models for the Cape Verde Rise [Courtney and White, 1986], the Bermuda Rise [Detrick et al., 1986], and the Hawaiian chain [Von Herzen et al., 1982, 1989]. The presence of large heat flow anomalies would argue for the existence of large-scale reheating.

Determination of the relative contributions of lithospheric thinning and dynamic support to the superswell is complicated by two additional effects. First, the bathymetry reflects the emplacement of the seamounts [McNutt and Fisher, 1987; McNutt and Judge, 1990]. In addition, the bathymetry may reflect anomalous conditions at the time when the lithosphere formed, prior to passage over the mantle region that gives rise to superswell phenomena. This possibility is suggested by the observation that 0 to $12-\mathrm{Ma}$ seafloor on the western flank of the East Pacific Rise is shallower and subsides more slowly than that on the eastern flank [Mammerickx et al., 1975; Cochran, 1986]. Marty and Cazenave [1989] determined similar slow subsidence rates for this area (region 27 in their study) extending from young lithosphere to $100-\mathrm{Ma}$ lithosphere. Hence Calmant et al. [1990] suggest that the regional depth anomalies may, in part, be remnants of the ridge processes, unrelated to midplate hot spot volcanism. Altematively, the difference in subsidence rates between the two sides of the East Pacific Rise may reflect mantle processes and flow (M. McNutt, personal communication, 1990). 
In this paper we examine the heat flow data for the South Pacific superswell region to determine if there is any evidence for higher than expected lithospheric temperatures. We then investigate whether a thinned lithosphere or reheating model can both explain the observed heat flow data and produce sufficiently high upper lithospheric temperatures to account for the lower lithospheric strength implied by the flexural data. In addition, we examine the heat flow for the Darwin Rise to determine if there is any detectable present temperature anomaly associated either with the mid-Cretaceous volcanic events or indicating if superswell conditions might still be present.

\section{Heat Flow Data SET}

We have compiled heat flow data for the Pacific plate using the data from Louden [1989] and published and unpublished data from Lamont-Doherty Geological Observatory (LDGO) (Figure 1). The youngest lithosphere included is $20 \mathrm{Ma}$, the age of the youngest available heat flow measurement site for the South Pacific superswell region. We excluded data within a few hundred kilometers of trenches, where bending of the lithosphere may cause fracturing and hence reintroduce water circulation in the crust [Abbott and Fisk, 1986]. Our data set contains 853 measurements, of which 124 are for the Darwin Rise and 76 are for the South Pacific superswell region. Of these, the unpublished LDGO data account for 21 from the superswell and 32 for the Darwin Rise. The heat flow data for the superswell and Darwin Rise are listed in Tables 1 and 2. In assembling the heat flow data, we considered a number of factors including the site sedimentary environment, thermal conductivity, and instrument evaluation.

\section{Sedimentary Environment}

Heat flow measurements can be biased in conditions where a significant portion of the heat transfer occurs via water circulation, rather than conduction in the rock and sediment [Lister. 1972; Williams et al., 1974]. As the sediment cover increases to a thickness of $100-200 \mathrm{~m}$ and insulates the convecting system, the average measured heat flow increases and approaches that theoretically expected by plate cooling models, which assume conductive heat transfer [Anderson and Hobart, 1976; Sclater et al., 1976; Davis and Lister, 1977]. Heat flow sites are thus characterized by the sedimentary cover to indicate whether water circulation may be affecting the heat flow.

We examined seismic reflection records for the LDGO heat flow sites to determine the sedimentary environment, using categories proposed by Sclater et al. [1976]. A site classified "A" has flat or rolling hills with more than 150-200 $\mathrm{m}$ of continuous sediment cover within $18 \mathrm{~km}$. A "B" site has flat or rolling hills with generally continuous sediment cover, with either an outcropping basement high or thin sedimentary cover. A "C" site is an area of rolling hills or rough topography with a thin or variable sediment cover, and " $D$ " denotes a site with sediment in ponds next to outcropping basement highs.

\section{Conductivity}

The heat flow is the product of the measured thermal gradient and the thermal conductivity. Although ideally the conductivity should be measured either in situ or from a piston core taken at the measurement site, approximately $28 \%$ of the heat flow measurements for the superswell and Darwin Rise and $41 \%$ of those for the rest of the Pacific plate have estimated conductivities. Often in earlier studies, the estimates were based on the nearest available conductivity measurement, at times $500 \mathrm{~km}$ or more away, or global average values.

Stein and Abbott [1991] examined methods to best estimate the thermal conductivity. They found that the variation between the closest adjacent sites with measured conductivity increases with increasing separation, such that sites within $200 \mathrm{~km}$ are on average significantly closer in conductivity than sites further apart. Two factors may contribute to this effect. Conductivity generally varies with lithology [e.g., Langseth and Von Herzen, 1970], with mean conductivity highest for carbonate oozes, intermediate for deep-sea clays, and least for siliceous oozes. Also, there is a suggestion that conductivity decreases with increasing seafloor depth. Tests with the measured data suggest that of several methods, the best predictor of conductivity is the mean value measured within $200 \mathrm{~km}$. Using the mean within a geographic region is a somewhat poorer estimate; and the ocean-wide mean and value at the nearest site are poorer still.

Following Stein and Abbott [1991], we reassigned the heat flow values with estimated conductivity in the superswell and Darwin Rise. Fourteen of the superswell and eight of the Darwin Rise values had measured conductivity within $200 \mathrm{~km}$. Nine of the superswell and 23 of the Darwin Rise values did not have measured conductivity within $200 \mathrm{~km}$ and new values were estimated from regional averages. The mean of the absolute difference between the old and new conductivity values was about $10 \%\left(0.073 \mathrm{~W} \mathrm{~m}^{-1} \mathrm{~K}^{-1}\right)$, with 35 of the 54 new values higher than the original estimate.

\section{Instrument Evaluations}

The quality of heat flow measurements are evaluated from the number of temperature measurements, the errors of the measurements at each depth, the tilt of the instrument, and whether or not conductivity was measured or estimated. The evaluation scale for LDGO data is from 10 (excellent) to 0 (poor) [Langseth and Taylor, 1967]. Louden [1989] uses a different scale, in which measurements are evaluated as A, B, C or E. For comparison, LDGO evaluations 9 and 10 would be A, 6-8 would be $B$, and 5-1 would be $C$. An $E$ evaluation means that no information is available on the quality.

Heat flow measurements with evaluations less than or equal to 5 have generally been considered unreliable. To test this, we grouped the Pacific plate data set by the evaluations, and examined its variation with age (Figure 3a). The lithospheric ages of the heat flow sites were determined by interpolating the isochrons of McNutt and Fisher [1987] for the superswell, Renkin and Sclater [1988] for the North Pacific, and S. Cande (personal communication, 1989) for the rest of the Pacific plate. The age bins used were those from the global compilation of Sclater et al. [1980]. The means, medians, and standard deviations for the data are plotted in Figure 3b. Except for the measurements with the poorest evaluations, the standard deviations for the data from older ( $>100 \mathrm{Ma}$ ) lithosphere are significantly less than for the younger lithosphere. The standard deviations for the poorest group ( $\leq 5$, or categories $C$ and $E$ ) generally exceed those in either the 9-10 (category A) or 6-8 (category B) group. This generalization fails only for three age bins (65-80 Ma, 125-140 $\mathrm{Ma}$ and $160-200 \mathrm{Ma}$ ) with only a small number of measurements $(5,4$, and 3 respectively) in the poorest group. Hence for our analysis of the superswell and Darwin Rise regions, we used only stations with evaluations in categories A and B (10-6).

For the measurements with the best evaluations (category A; 9-10) the median in each age bin is almost the same as the mean. In general, the difference between the mean and the 
Table 1. Superswell Region

\begin{tabular}{|c|c|c|c|c|c|c|c|c|c|}
\hline Cruise/No. & Area $^{a}$ & $\begin{array}{c}\text { Latitude, } \\
\text { deg }\end{array}$ & $\begin{array}{c}\text { Longitude, } \\
\text { deg }\end{array}$ & $\begin{array}{l}\text { Heat Flow, } \\
\mathrm{mW} \mathrm{m}^{-2}\end{array}$ & Evaluation ${ }^{b}$ & Environment ${ }^{f}$ & $\begin{array}{c}\text { Depth, } \\
\text { m }\end{array}$ & Reference $^{d}$ & Notes $^{c}$ \\
\hline \multicolumn{10}{|c|}{ Unpublished Data } \\
\hline C11-114 & NF & -6.13 & -140.85 & 58.6 & 9 & $\mathbf{A}$ & 4343 & 1 & \\
\hline C11-116 & NF & -10.83 & -143.97 & 57.4 & 9 & D & 4749 & 1 & \\
\hline C11-117 & NF & -12.87 & -145.55 & 57.4 & 8 & C & 4662 & 1 & \\
\hline C11-119 & SS & -14.82 & -139.97 & 55.3 & 10 & $\mathrm{C}$ & 2950 & 1 & \\
\hline C11-120 & SS & -12.32 & -136.95 & 42.3 & 7 & D & 4103 & 1 & \\
\hline C11-121 & NF & -10.63 & -134.87 & 28.1 & 7 & C & 3860 & 1 & \\
\hline C15-4 & SF & -26.78 & -135.40 & 745. & 3 & D & 4248 & 1 & \\
\hline C15-6 & SF & -27.08 & -129.40 & 14.2 & 6 & C & 3871 & 1 & \\
\hline C17-130 & SF & -25.22 & -159.60 & 23.2 & 10 & D & 5231 & 1 & \\
\hline C17-132 & SS & -22.88 & -157.97 & 64.6 & 10 & D & 4759 & 1 & \\
\hline C17-133 & SS & -23.12 & -154.70 & 67.5 & 10 & D & 4869 & 1 & \\
\hline V21-49 & SS & -14.88 & -135.05 & 38.7 & 4 & $\mathrm{C}$ & 4431 & 1 & \\
\hline ARIAD-2 & SS & -18.76 & -129.77 & 63.7 & 7 & B & 4130 & 2 & $A, G$ \\
\hline ARIAD-4A & SS & -18.81 & -129.77 & 127. & 8 & B & 4150 & 2 & $A, G$ \\
\hline ARIAD-4B & SS & -18.81 & -129.77 & 88.5 & 8 & B & 4130 & 2 & $A, G$ \\
\hline ARIAD-4C & SS & -18.81 & -129.76 & 83.1 & 8 & B & 4130 & 2 & $A, G$ \\
\hline ARIAD-4E & SS & -18.81 & -129.76 & 63.7 & 8 & B & 4150 & 2 & $A, G$ \\
\hline ARIAD-4F & SS & -18.81 & -129.75 & 76.6 & 8 & B & 4170 & 2 & $A, G$ \\
\hline ARIAD-4G & SS & -18.80 & -129.74 & 56.1 & 8 & B & 4170 & 2 & A,G \\
\hline ARIAD-4H & SS & -18.80 & -129.74 & 61.5 & 8 & B & 4170 & 2 & $A, G$ \\
\hline ARIAD-4I & SS & -18.80 & -129.74 & 52.9 & 8 & B & 4170 & 2 & $A, G$ \\
\hline
\end{tabular}

\begin{tabular}{|c|c|c|c|c|c|c|c|c|c|}
\hline Cruise/No. & Area $^{a}$ & $\begin{array}{c}\begin{array}{c}\text { Latitude, } \\
\text { deg }\end{array} \\
\end{array}$ & $\begin{array}{c}\begin{array}{c}\text { Longitude, } \\
\text { deg }\end{array} \\
\end{array}$ & $\begin{array}{l}\text { Heat Flow, } \\
\mathrm{mW} \mathrm{m}^{-2}\end{array}$ & Evaluation $^{b}$ & Environment ${ }^{t}$ & $\begin{array}{c}\text { Depth, } \\
\text { m }\end{array}$ & Reference $^{d}$ & Notes $^{6}$ \\
\hline \multicolumn{10}{|c|}{ Published Data } \\
\hline CAP-31B & SS & -17.47 & -158.67 & 66.2 & A & $\mathrm{U}$ & 4880 & 3 & \\
\hline CAP-33B & SS & -12.80 & -143.55 & 15.1 & $\mathbf{A}$ & $\mathrm{U}$ & 4300 & 3 & \\
\hline D-2 & SS & -14.98 & -136.02 & 27.2 & $\mathbf{E}$ & $\mathrm{U}$ & 4510 & 4 & \\
\hline D-3 & SS & -21.67 & -147.68 & 40.6 & A & $\mathrm{U}$ & 4760 & 4 & \\
\hline MSN-64 & NF & -10.57 & -151.08 & 49.4 & A & U & 5070 & 5 & \\
\hline MSN-65 & NF & -8.28 & -151.60 & 60.3 & A & $\mathrm{U}$ & 5190 & 5 & \\
\hline MSN-66 & NF & -5.92 & -149.65 & 31.4 & A & $\mathrm{U}$ & 5160 & 5 & \\
\hline MSN6-62 & $\mathrm{SF}$ & -27.32 & -157.50 & 44.4 & A & A & 4960 & 6 & \\
\hline RIS- 120 & SS & -13.87 & -125.33 & 43.5 & $\mathbf{A}$ & $\mathrm{U}$ & 3680 & 5 & \\
\hline RIS-121 & SS & -14.03 & -127.12 & 7.5 & B & $\mathrm{U}$ & 3930 & 5 & L \\
\hline RIS-122 & SS & -14.03 & -128.42 & 42.7 & A & $\mathrm{U}$ & 3995 & 5 & \\
\hline RIS-123 & SS & -14.03 & -129.80 & 119. & B & $\mathrm{U}$ & 4120 & 5 & L \\
\hline RIS-124 & SS & -14.05 & -130.30 & 34.3 & B & $\mathrm{U}$ & 4090 & 5 & $\mathrm{~L}$ \\
\hline RIS-125 & SS & -14.05 & -131.73 & 20.1 & $\overline{\mathbf{A}}$ & $\mathrm{U}$ & 4010 & 5 & \\
\hline RIS-127 & SS & -14.03 & -133.75 & 41.6 & B & $\mathrm{U}$ & 4290 & 5 & $\mathrm{~L}$ \\
\hline RIS-128 & SS & -14.03 & -134.92 & 49.0 & $\bar{A}$ & $\mathbf{U}$ & 4220 & 5 & \\
\hline RIS-129 & SS & -14.05 & -136.57 & 29.3 & B & $\mathbf{U}$ & 4290 & 5 & $\mathbf{L}$ \\
\hline RIS-130 & SS & -14.15 & -138.10 & 71.2 & A & $\mathrm{U}$ & 4040 & 5 & \\
\hline RIS-131 & SS & -14.05 & -139.58 & 69.9 & A & $\mathbf{U}$ & 3925 & 5 & \\
\hline RIS-132 & SS & -14.92 & -141.57 & 78.9 & B & $\bar{U}$ & 2610 & 5 & L \\
\hline RIS-133 & SS & -15.25 & -142.43 & 46.9 & A & $\mathrm{U}$ & 3725 & 5 & \\
\hline RIS-134 & SS & -16.50 & -145.12 & 71.2 & $\mathrm{E}$ & $\mathrm{U}$ & 1440 & 5 & \\
\hline RIS-135 & SS & -16.87 & -145.82 & 56.5 & $\mathbf{A}$ & $\mathbf{U}$ & 2750 & 5 & \\
\hline RIS-136 & SS & -17.08 & -147.22 & 8.8 & A & $\mathbf{U}$ & 4190 & 5 & \\
\hline RIS-137 & SS & -16.77 & -148.87 & 6.6 & B & $\mathbf{U}$ & 4200 & 5 & L \\
\hline RIS- 138 & SS & -16.57 & -148.50 & 47.3 & $\mathbf{A}$ & $\mathbf{U}$ & 4250 & 5 & \\
\hline RIS-140 & SS & -14.72 & -145.67 & 50.2 & $\mathbf{A}$ & $\mathrm{U}$ & 2770 & 5 & \\
\hline RIS-14i & SS & -13.62 & -145.05 & 12.1 & B & $\mathrm{U}$ & 4390 & 5 & \\
\hline RIS-142 & SS & -13.05 & -144.05 & 54.0 & $\mathbf{A}$ & $\mathbf{U}$ & 4960 & 5 & \\
\hline RIS-143 & SS & -12.77 & -143.57 & 49.1 & B & $\mathbf{U}$ & 4480 & 5 & L \\
\hline RIS-144 & $\mathrm{NF}$ & -11.97 & -142.45 & 49.8 & $\mathbf{A}$ & $\mathbf{U}$ & 4520 & 5 & \\
\hline RIS-145 & $\mathrm{NF}$ & -11.08 & -140.95 & 17.0 & B & $\mathrm{U}$ & 4270 & 5 & $\mathrm{~L}$ \\
\hline RIS-146 & NF & -10.50 & -139.98 & 15.5 & $\mathbf{A}$ & $\overline{\mathbf{U}}$ & 4140 & 5 & \\
\hline RIS-147 & $\mathrm{NF}$ & -8.63 & -138.30 & 69.9 & A & $\mathbf{U}$ & 4080 & 5 & \\
\hline RIS-148 & NF & -7.45 & -137.18 & 32.7 & $\mathrm{E}$ & $\mathbf{U}$ & 4400 & 5 & L \\
\hline RIS-149 & NF & -6.38 & -136.18 & 54.9 & A & $\mathbf{U}$ & 4350 & 5 & \\
\hline SCAN8-H1 & NF & -6.56 & -136.01 & 108. & A & $\mathbf{U}$ & 4440 & 7 & L \\
\hline SCAN8-H2 & SS & -12.24 & -134.31 & 86.7 & A & $\mathbf{U}$ & 4225 & 7 & \\
\hline SCAN8-H4 & SF & -24.89 & -143.49 & 56.4 & A & $\mathbf{U}$ & 4624 & 7 & $\mathrm{~L}$ \\
\hline ST8HF48 & SS & -12.58 & -125.99 & 23.0 & $\mathrm{E}$ & C & 4015 & 8 & L \\
\hline ST8HF49 & SS & -14.01 & -134.06 & 59.2 & $\mathbf{E}$ & B & 4469 & 8 & $\mathrm{~L}$ \\
\hline C15-2 & SF & -23.50 & -141.15 & 107. & 7 & D & 4546 & 8 & \\
\hline C15-3 & SF & -25.12 & -138.43 & 189. & 4 & $\mathrm{D}$ & 4212 & 8 & \\
\hline C15-5 & SF & -27.07 & -129.20 & 18.0 & 6 & C & 3860 & 8 & \\
\hline C15-7 & SF & -27.13 & -128.30 & 62.0 & 8 & C & 3977 & 8 & \\
\hline C15-8 & $S F$ & -27.33 & -127.85 & 84.6 & 6 & D & 3720 & 8 & \\
\hline V18-100 & NF & -9.70 & -136.47 & 69.3 & 10 & B & 4329 & 9 & \\
\hline V18-101 & $\mathrm{NF}$ & -8.00 & -133.83 & 80.9 & 10 & B & 4565 & 9 & \\
\hline
\end{tabular}


TABLE 1. (continued)

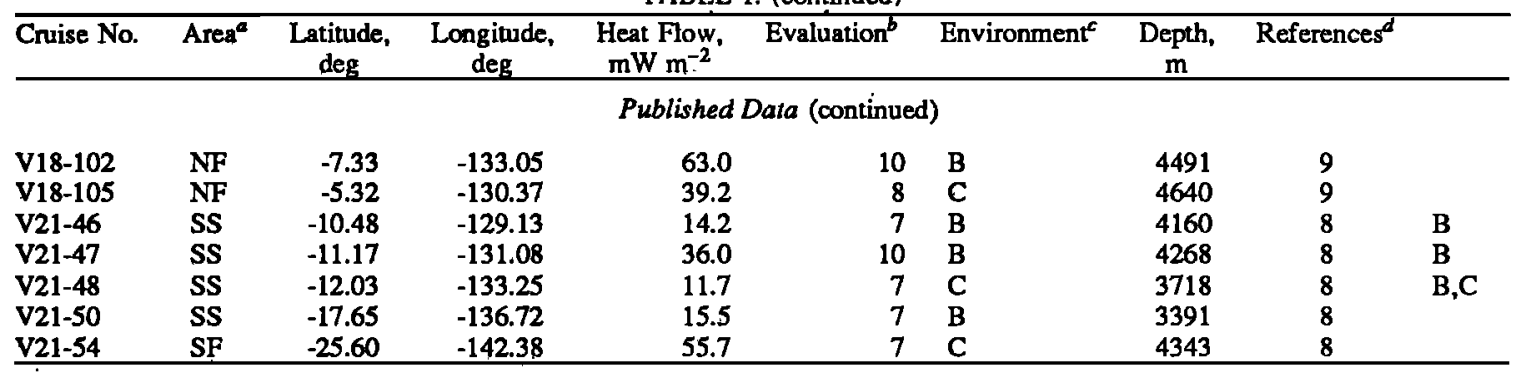

areas are as follows: NF, north of Marquesas fracture zone; SF, south of Austral fracture zone; SS, between Marquesas and Austral fracture zones.

beasurement evaluation. Scales are 0-10 from Langseth and Taylor [1967] and A-E from Louden [1989].

"Sedimentary environment from Sclater et al. [1976]. "U" denotes sites with undetermined environments.

References are as follows: 1, unpublished LDGO data; 2, M. A. Hobart et al., manuscript in preparation, 1991; 3 , Maxwell [1958]; 4, Von Herzen [1959]; 5, Von Herzen and Uyeda [1963]; 6, Anderson and Von Herzen [1978]; 7, Sclater et al. [1972]; 8, Anderson et al. [1978]; 9, Langseth et al. [1965].

Notes are as follows: A, M. A. Hobart determined a thermal conductivity of $0.77 \mathrm{~W} \mathrm{~m}^{-1} \mathrm{~K}^{-1}$ for ARIAD data using the water content method. Following Stein and Abbott [1991], we reestimate the thermal conductivity at $0.831 \mathrm{~W} \mathrm{~m}^{-1} \mathrm{~K}^{-1}$. B, reevaluated environment. $C$, typographical error in published heat flow value. $G$, conductivity reestimated using regional method. L, conductivity reestimated using local method.

Table 2. Darwin Rise

\begin{tabular}{|c|c|c|c|c|c|c|c|c|}
\hline Cruise/No. & $\begin{array}{c}\text { Latitude, } \\
\text { deg }\end{array}$ & $\begin{array}{c}\text { Longitude, } \\
\text { deg }\end{array}$ & $\begin{array}{l}\text { Heat Flow, } \\
\text { mW m }^{-2}\end{array}$ & Evaluation $^{a}$ & Environment $^{b}$ & $\begin{array}{c}\text { Depth, } \\
\text { m }\end{array}$ & Reference $^{c}$ & Note $^{d}$ \\
\hline \multicolumn{9}{|c|}{ Unpublished Dala } \\
\hline $\mathrm{C} 10-36$ & 2.05 & -169.85 & 50.2 & 10 & B & 5365 & 1 & \\
\hline C10-37 & 4.90 & -171.42 & 86.4 & 4 & B & 5411 & 1 & G \\
\hline C10-38 & 7.52 & -172.83 & 54.5 & 8 & C & 5865 & 1 & $\mathbf{G}$ \\
\hline C10-41 & -1.65 & 178.18 & 54.0 & 10 & C & 5440 & 1 & \\
\hline $\mathrm{C} 10-42$ & -3.97 & 176.05 & 34.2 & 6 & C & 5026 & 1 & G \\
\hline C10-67 & 16.90 & 155.13 & 60.7 & 10 & $\mathbf{A}$ & 5775 & 1 & \\
\hline C10-68 & 19.45 & 156.42 & 32.5 & 4 & B & 5447 & 1 & G \\
\hline C10-69 & 22.35 & 157.82 & 58.2 & 8 & $\mathbf{A}$ & 5402 & 1 & \\
\hline C10-70 & 24.78 & 159.13 & 48.2 & 4 & B & 5682 & 1 & G \\
\hline C12-40 & 3.68 & -165.05 & 59.3 & 10 & $\mathbf{A}$ & 5351 & 1 & \\
\hline C12-41 & 2.33 & -165.20 & 59.8 & 10 & $\mathrm{~B}$ & 5365 & 1 & \\
\hline $\mathrm{C} 12-42$ & 0.65 & -164.87 & 49.4 & 10 & B & 5106 & 1 & \\
\hline $\mathrm{Cl} 2-43$ & -0.18 & -164.50 & 53.8 & 8 & D & 5336 & 1 & \\
\hline C12-44 & -4.55 & -163.28 & 48.4 & 9 & C & 4839 & 1 & \\
\hline C12-45 & -7.73 & -162.38 & 9.6 & 6 & A & 4266 & 1 & \\
\hline C12-66 & 12.48 & 157.00 & 51.5 & 8 & B & 5883 & 1 & \\
\hline Ci7-118 & 4.10 & 165.73 & 50.3 & 10 & $\mathbf{A}$ & 4574 & 1 & \\
\hline C17-119 & 6.80 & 165.40 & 55.0 & 10 & A & 5158 & $i$ & \\
\hline C17-120 & 4.63 & 166.83 & 52.3 & 10 & $\mathbf{A}$ & 4729 & 1 & \\
\hline C17-121 & 3.50 & 168.83 & 58.0 & 10 & $\mathbf{A}$ & 4376 & 1 & \\
\hline C17-122 & 4.18 & 173.50 & 95.6 & 8 & A & 4900 & 1 & \\
\hline V21-63 & 26.43 & 169.03 & 53.6 & 10 & B & 5982 & 1 & \\
\hline V21-64 & 27.08 & 166.07 & 51.5 & 10 & $\mathbf{A}$ & 5954 & 1 & \\
\hline V21-65 & 27.90 & 162.52 & 50.2 & 10 & B & 5954 & 1 & \\
\hline V24-64 & 11.85 & 169.68 & 47.7 & 4 & $\mathbf{A}$ & 5137 & 1 & \\
\hline V28-110 & 15.68 & -165.22 & 24.0 & 6 & B & 5320 & 1 & \\
\hline V28-112 & 10.65 & -169.80 & 17.6 & 9 & $C$ & 2439 & 1 & \\
\hline V28-113 & 7.67 & -171.75 & 42.3 & 10 & B & 5404 & 1 & \\
\hline V32-40 & 28.42 & 159.13 & 47.8 & 10 & B & 6030 & 1 & \\
\hline V32-41 & 25.93 & 156.17 & 52.2 & 10 & $\vec{A}$ & 5961 & 1 & \\
\hline V32-70 & 17.20 & 156.37 & 53.2 & 8 & $\mathbf{A}$ & 5809 & 1 & \\
\hline V32-71 & 2.30 & 167.10 & 49.9 & 10 & A & 4287 & 1 & \\
\hline
\end{tabular}

\begin{tabular}{|c|c|c|c|c|c|c|c|c|}
\hline Cruise/No. & $\begin{array}{c}\text { Latitude, } \\
\text { deg }\end{array}$ & $\begin{array}{c}\text { Longitude, } \\
\text { deg }\end{array}$ & $\begin{array}{l}\text { Heat Flow, } \\
\mathrm{mW} \mathrm{m}^{-2}\end{array}$ & Evaluation $^{a}$ & Environment $^{b}$ & $\begin{array}{c}\text { Depth, } \\
\text { m }\end{array}$ & Reference $^{c}$ & Note $^{d}$ \\
\hline \multicolumn{9}{|c|}{ Published Data } \\
\hline$V 24-51$ & 9.30 & -155.52 & 43.5 & 7 & $\mathbf{A}$ & 5234 & 10 & \\
\hline V24-52 & 10.22 & -155.02 & 138. & 4 & $\mathbf{A}$ & 5302 & 10 & \\
\hline V24-53 & 11.77 & -154.03 & 53.2 & 6 & A & 5209 & 10 & \\
\hline V24-54 & 13.13 & -154.53 & 44.0 & 10 & B & 5451 & 10 & \\
\hline$v 24-55$ & 14.95 & -155.30 & 58.2 & 8 & $\mathbf{A}$ & 5539 & 10 & \\
\hline V24-57 & 17.52 & -164.68 & 48.6 & 8 & A & 5473 & 10 & \\
\hline V24-58 & 16.48 & -166.78 & 54.8 & 10 & $\mathbf{A}$ & 5234 & 10 & \\
\hline V24-59 & 13.30 & -172.92 & 58.6 & 8 & B & 5680 & 10 & \\
\hline V24-60 & 12.02 & -175.62 & 67.4 & 8 & $\ddot{\mathbf{B}}$ & 5280 & 10 & \\
\hline V24-62 & 9.28 & 178.95 & 72.4 & 10 & C & 5704 & 10 & \\
\hline V24-63 & 10.88 & 173.00 & 57.8 & 10 & $\mathbf{A}$ & 5374 & 10 & \\
\hline
\end{tabular}


Table 2. (continued)

\begin{tabular}{|c|c|c|c|c|c|c|c|c|}
\hline Cruise No. & $\begin{array}{c}\text { Latitude, } \\
\text { deg }\end{array}$ & $\begin{array}{c}\text { Longitude, } \\
\text { deg }\end{array}$ & $\begin{array}{l}\text { Heat Flow, } \\
\mathrm{mW} \mathrm{m}^{-2}\end{array}$ & Evaluation $^{a}$ & Environment $^{b}$ & $\begin{array}{c}\text { Depth, } \\
\text { m }\end{array}$ & References $^{c}$ & Note $^{d}$ \\
\hline \multicolumn{9}{|c|}{ Published Dafa (continued) } \\
\hline V24-65 & 14.13 & 166.80 & 64.9 & 8 & $\mathbf{A}$ & 5587 & 10 & \\
\hline V24-67 & 20.87 & 165.12 & 49.8 & 8 & A & 5544 & 10 & \\
\hline V24-69 & 23.65 & 170.87 & 51.9 & 8 & A & 5936 & 10 & \\
\hline V24-76 & 21.78 & -178.78 & 65.1 & 8 & B & 5444 & 10 & G \\
\hline V24-77 & 16.13 & 179.73 & 55.3 & 10 & A & 5330 & 10 & \\
\hline V24-79 & 8.32 & 176.42 & 52.2 & 4 & C & 5097 & 10 & \\
\hline V24-80 & 6.57 & 173.50 & 73.4 & 8 & B & 4991 & 10 & \\
\hline V24-81 & 4.85 & 170.92 & 47.4 & 10 & A & 4501 & 10 & \\
\hline V24-82 & 2.82 & 168.18 & 61.3 & 8 & A & 4409 & 10 & $\mathrm{~L}$ \\
\hline CAP-2B & 0.67 & 169.28 & 78.7 & A & $\mathrm{U}$ & 4310 & 3 & \\
\hline CIRCE-6H & 21.62 & 168.48 & 42.5 & A & $\mathrm{U}$ & 5698 & 7 & $\mathrm{~L}$ \\
\hline CIRCE-7H & 18.45 & 159.82 & 46.2 & A & $\mathrm{U}$ & 5668 & 7 & G \\
\hline DSDP462A & 7.24 & 165.03 & 49.0 & A & A & 5181 & 14 & \\
\hline LSDH-60 & 6.28 & 168.18 & 41.0 & A & $\mathrm{U}$ & 4840 & 11 & \\
\hline LSDH-61 & 7.42 & 167.92 & 44.8 & A & $\mathrm{U}$ & 4950 & 11 & \\
\hline LSDH-62 & 10.92 & 174.02 & 58.2 & A & $\mathrm{U}$ & 5535 & 11 & \\
\hline LSDH-63 & 8.15 & -177.13 & 50.7 & A & $\mathrm{U}$ & 5310 & 11 & \\
\hline LSDH-64 & 9.40 & -171.40 & 31.7 & B & $\mathrm{U}$ & 4210 & 11 & G \\
\hline LSDH-66 & 10.65 & -169.75 & 27.4 & B & $\mathrm{U}$ & 2500 & 11 & $\mathbf{L}$ \\
\hline LSDH-67 & 11.45 & -168.83 & 47.7 & A & $\mathrm{U}$ & 5290 & 11 & \\
\hline МАHI-47 & -9.20 & -173.98 & 81.2 & A & $\mathrm{U}$ & 5227 & 11 & \\
\hline MAHI-49 & -3.83 & -169.07 & 69.5 & B & $\mathrm{U}$ & 5020 & 11 & \\
\hline MAHI-50 & -0.57 & -167.60 & 45.6 & A & $\mathrm{U}$ & 5355 & 11 & \\
\hline MAHI-51 & -1.57 & -167.72 & 52.3 & A & $\mathrm{U}$ & 5683 & 11 & \\
\hline MP-32 & 18.30 & -173.38 & 30.2 & A & U & 3900 & 3 & \\
\hline MP-35-2 & 19.47 & -174.58 & 54.0 & A & $\mathrm{U}$ & 4900 & 3 & \\
\hline MP-36 & 16.75 & -176.40 & 49.8 & A & $\mathrm{U}$ & 5040 & 3 & \\
\hline MP-38 & 19.03 & -177.32 & 45.6 & $\ddot{A}$ & $\mathrm{U}$ & 4750 & 3 & \\
\hline MSN-5 & 14.18 & -161.13 & 46.5 & $\ddot{A}$ & $\mathrm{U}$ & 5685 & 11 & \\
\hline MSN-6 & 7.63 & -168.12 & 54.0 & $\mathbf{A}$ & $\mathrm{U}$ & 5000 & 11 & \\
\hline MSN-7 & 3.10 & -173.97 & 51.5 & A & $\mathrm{U}$ & 5250 & 11 & \\
\hline MSN-8 & 0.10 & 179.95 & 56.9 & A & $\mathrm{U}$ & 5290 & 11 & \\
\hline NOVA-A3 & 18.72 & -167.78 & 57.3 & A & U & 5021 & 7 & G \\
\hline NOVA-A4 & 18.15 & -170.43 & 74.5 & B & $\mathrm{U}$ & 5198 & 7 & \\
\hline NOVA-A5 & 17.25 & -173.85 & 46.3 & B & $\mathrm{U}$ & 5059 & 7 & L \\
\hline NOVA-A6 & 16.58 & -176.52 & 54.3 & $\tilde{A}$ & $\mathrm{U}$ & 5199 & 7 & $\bar{L}$ \\
\hline NOVA-A7 & 16.00 & -179.10 & 46.8 & A & $\mathrm{U}$ & 5094 & 7 & L \\
\hline NOVA-A8 & 14.03 & -179.23 & 48.2 & A & $\mathrm{U}$ & 5639 & 7 & G \\
\hline NOVA-A9 & 11.82 & -179.10 & 44.4 & A & $\mathrm{U}$ & 5540 & 7 & $\mathbf{G}$ \\
\hline NOVA-A10 & 9.90 & -179.00 & 55.3 & A & U & 6148 & 7 & \\
\hline NOVA-A11 & 8.12 & -179.12 & 54.3 & A & $\mathrm{U}$ & 5740 & 7 & L \\
\hline NOVA-A12 & 6.00 & -179.00 & 45.8 & A & $\mathrm{U}$ & 5778 & 7 & $\mathbf{G}$ \\
\hline NOVA-A13 & 3.93 & -178.78 & 49.0 & A & $\mathrm{U}$ & 5384 & 7 & \\
\hline NOVA-A15 & -0.02 & -179.13 & 56.5 & $\mathbf{A}$ & $\mathrm{U}$ & 5404 & 7 & \\
\hline NOVA-A16 & -1.98 & -179.02 & 54.0 & A & $\tilde{U}$ & 5584 & 7 & \\
\hline NOVA-A17 & -4.02 & -178.75 & 62.2 & A & $\mathrm{U}$ & 5989 & 7 & G \\
\hline NOVA-A18 & -7.67 & -178.60 & 67.6 & $\mathbf{A}$ & $\mathrm{U}$ & 6018 & 7 & G \\
\hline PROA-40 & -3.12 & 174.42 & 40.6 & A & $\mathrm{U}$ & 4950 & 11 & \\
\hline PROA-41 & -2.07 & 172.48 & 33.5 & A & U & 4270 & 11 & \\
\hline PROA-44 & 3.67 & -176.25 & 18.4 & B & $\mathrm{U}$ & 4995 & 11 & \\
\hline PROA-45 & 1.95 & -178.02 & 56.3 & B & $\mathrm{U}$ & 5330 & 11 & G \\
\hline PROA-46 & 0.25 & -179.70 & 58.6 & B & U & 5400 & 11 & \\
\hline PROA-47 & -1.38 & 178.40 & 53.2 & $\bar{A}$ & $\mathrm{U}$ & 5400 & 11 & \\
\hline PROA-54 & -4.05 & -165.42 & 22.6 & A & $\mathrm{U}$ & 4020 & 11 & \\
\hline PROA-55 & 6.38 & -172.53 & 49.8 & B & $\mathrm{U}$ & 5840 & 11 & G \\
\hline PROA-56 & 8.87 & -169.53 & 54.4 & $\vec{A}$ & $\mathrm{U}$ & 5300 & 11 & \\
\hline PROA-57 & 9.98 & -167.08 & 47.3 & A & $\mathrm{U}$ & 5250 & 11 & \\
\hline PROA-58 & 10.55 & -165.53 & 47.3 & $\ddot{A}$ & $\mathrm{U}$ & 4340 & 11 & \\
\hline SCAN3-H1 & 16.40 & -164.41 & 54.0 & $\ddot{A}$ & $\mathrm{U}$ & 5582 & 7 & \\
\hline SCAN5-H3 & 9.49 & 166.54 & 49.4 & $\mathbf{A}$ & $\mathrm{U}$ & 4592 & 7 & \\
\hline SCAN5-H4 & 9.34 & 179.90 & 38.9 & A & $\mathrm{U}$ & 6152 & 7 & \\
\hline SOLIS-22 & -4.78 & -157.95 & 25.9 & C & $\mathrm{U}$ & 1695 & 11 & G \\
\hline SOLIS-28 & -1.28 & -160.17 & 57.4 & B & $\mathbf{U}$ & 2532 & 11 & \\
\hline SOLIS-29 & -1.62 & -158.78 & 83.7 & B & $\mathbf{U}$ & 2015 & 11 & \\
\hline SOLIS-31 & -4.73 & -155.13 & 33.2 & $\mathbf{E}$ & $\mathbf{U}$ & 2538 & 11 & G \\
\hline SOLIS-41 & 0.60 & -172.22 & 46.5 & $\mathbf{A}$ & $\mathbf{U}$ & 5658 & 11 & \\
\hline SOLIS-43 & 2.22 & -166.45 & 50.7 & B & $\mathrm{U}$ & 5548 & 11 & \\
\hline SOLIS-44 & 8.75 & -160.53 & 18.0 & B & $\mathbf{U}$ & 4816 & 11 & \\
\hline SOLIS-45 & 7.68 & -164.80 & 46.9 & A & $\mathbf{U}$ & 4960 & 11 & \\
\hline SOLIS-47 & 5.43 & -173.10 & 47.7 & A & $\mathbf{U}$ & 5797 & 11 & \\
\hline SOLIS-48 & 4.20 & -178.07 & 46.8 & $\mathbf{E}$ & $\mathbf{U}$ & 5209 & 11 & G \\
\hline SOLIS-49 & 3.92 & -179.55 & 44.0 & $\mathbf{A}$ & $\mathbf{U}$ & 5684 & 11 & \\
\hline SOLIS-50 & 4.75 & 175.28 & 45.6 & A & $\mathbf{U}$ & 5122 & 11 & \\
\hline
\end{tabular}


Table 2. (continued)

\begin{tabular}{|c|c|c|c|c|c|c|c|c|}
\hline Cruise No. & $\begin{array}{c}\text { Latitude, } \\
\text { deg }\end{array}$ & $\begin{array}{c}\text { Longitude, } \\
\text { deg }\end{array}$ & $\begin{array}{l}\text { Heat Flow, } \\
\mathrm{mW} \mathrm{m}^{-2}\end{array}$ & Evaluation ${ }^{a}$ & Environment ${ }^{b}$ & $\begin{array}{c}\text { Depth, } \\
\text { m }\end{array}$ & References $^{c}$ & Note $^{d}$ \\
\hline \multicolumn{9}{|c|}{ Published Dala (continued) } \\
\hline SOLIS-51 & 6.17 & 173.00 & 9.6 & C & $\mathrm{U}$ & 4912 & 11 & \\
\hline V6334 & 11.10 & 159.00 & 58.8 & B & $\mathrm{U}$ & 5300 & 12 & G \\
\hline V6333-22 & 12.99 & 160.55 & 71.3 & B & $\mathrm{U}$ & 5140 & 12 & $\mathbf{G}$ \\
\hline V6333-25 & 12.89 & 160.71 & 186. & B & $\mathrm{U}$ & 4680 & 12 & $\mathrm{G}$ \\
\hline V6333-27 & 12.78 & 160.91 & 147. & B & $\mathrm{U}$ & 4640 & 12 & $\mathbf{G}$ \\
\hline ZETES-71 & 25.25 & 164.13 & 50.7 & $\mathbf{A}$ & $\mathrm{U}$ & 4943 & 13 & \\
\hline ZETES-72 & 24.89 & 163.35 & 47.3 & A & $\mathrm{U}$ & 4970 & 13 & \\
\hline ZETES-73 & 24.53 & 162.61 & 45.0 & B & $\mathrm{U}$ & 4930 & 13 & $\mathbf{L}$ \\
\hline
\end{tabular}

"Measurement evaluation. Scales are 0-10 from Langseth and Taylor [1967] and A-E from Louden [1989].

bSedimentary environment from Sclater et al. [1976]. "U" denotes sites with undetermined environments.

'References are as follows: 1, unpublished LDGO data; 3, Maxwell [1958]; 7, Sclater et al. [1972]; 10, Langseth et al. [1971]; 11, Halunen and Von Herzen [1973]; 12, Kuzmin et al. [1972]; 13, Vacquier et al. [1966]; 14, Boyce [1981].

Notes are as follows: $G$, conductivity reestimated using regional method. L. Conductivity reestimated using local method.
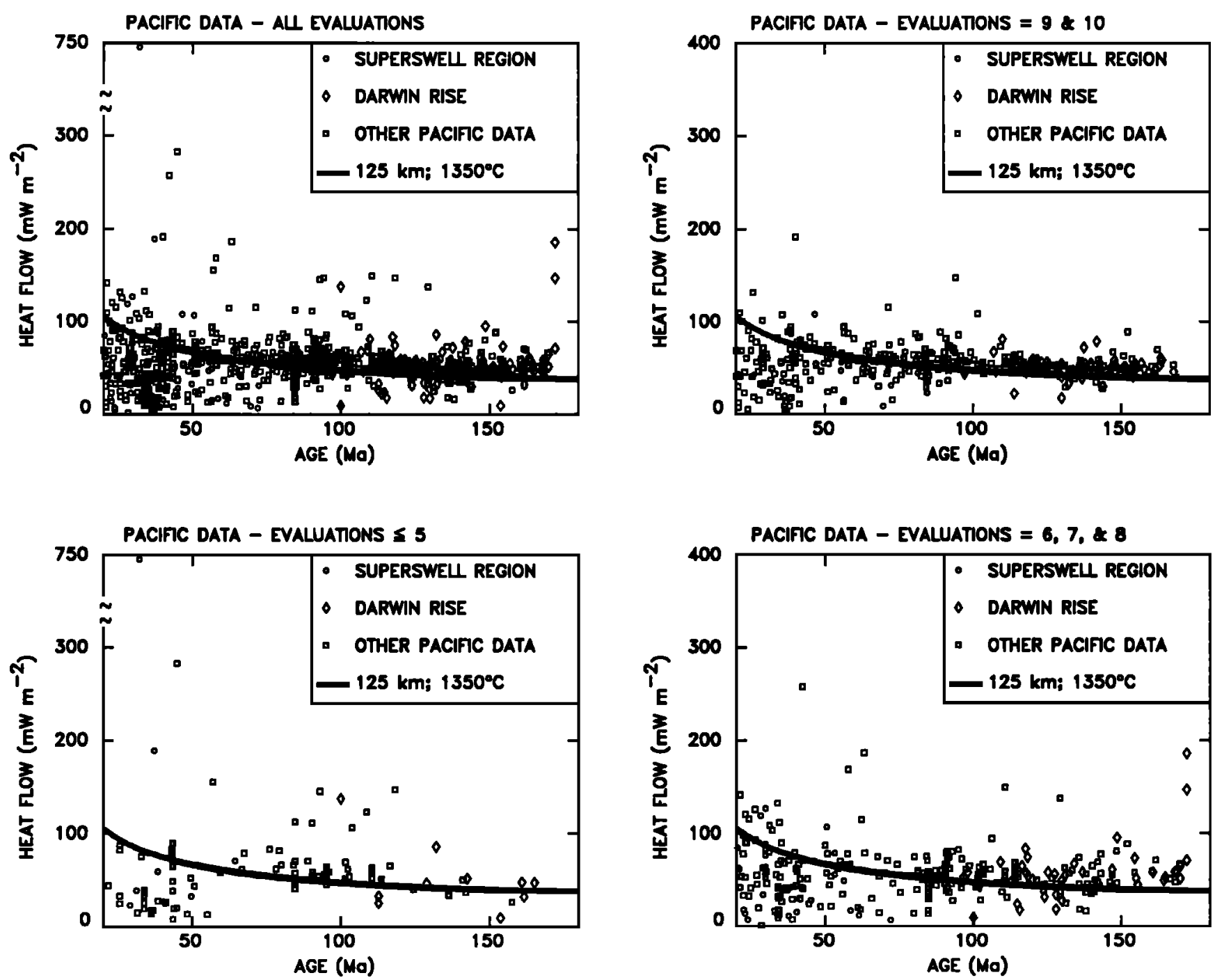

Fig. 3a. Heat flow data for the Pacific plate (Figure 1) as a function of age, sorted by instrument evaluations. LDGO data are evaluated from 10 (excellent) to 0 . The measurements with the poorest evaluations ( $\leq 5$; bottom left) have considerably more scatter than those with the better (6-8) and best $(9-10)$ evaluations plotted on the right side. The thick solid lines show the heat flow predicted for a $125-\mathrm{km}$-thick plate with a $1350^{\circ} \mathrm{C}$ basal temperature.

median decreases with increasing age and is most noticeable for the poorest evaluation category. Presumably, this variation reflects the trend of lower standard deviations with both increasing age and instrument evaluations.

We compared the variation in heat flow with age to that predicted by a standard thermal model of a $125-\mathrm{km}$-thick plate with a basal temperature of $1350^{\circ} \mathrm{C}$ [Parsons and Sclater, 1977]. Over the 20- to $180-\mathrm{Ma}$ age range, neither the entire heat flow data nor those with only the best $(9-10)$ evaluations show convincing evidence for a decrease in heat flow with age. For ages younger than about $50 \mathrm{Ma}$ the measured heat flow is less than predicted, presumably because much of the heat 
PACIFIC DATA - ALI EVALUATIONS
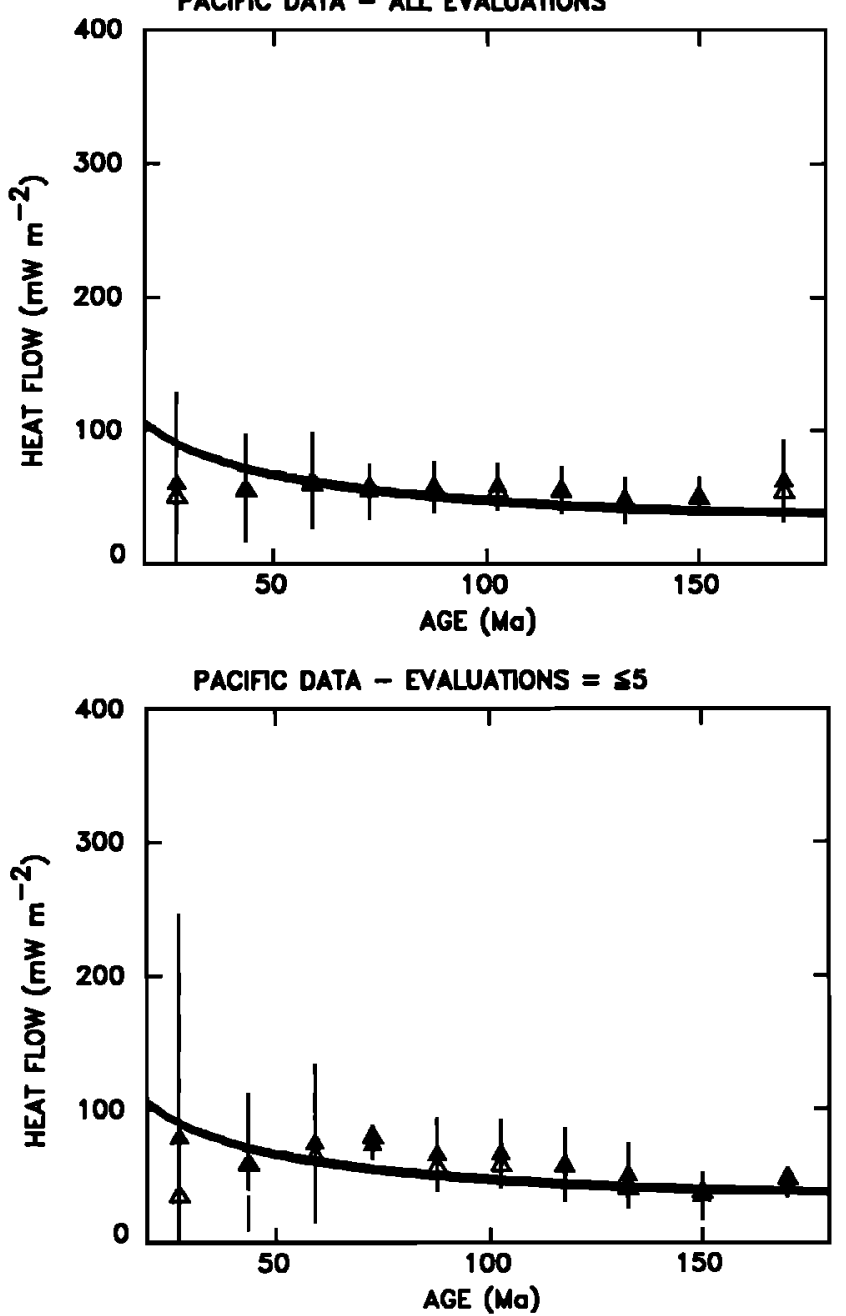

PACIFIC DATA - EVALUATIONS $=9 \& 10$
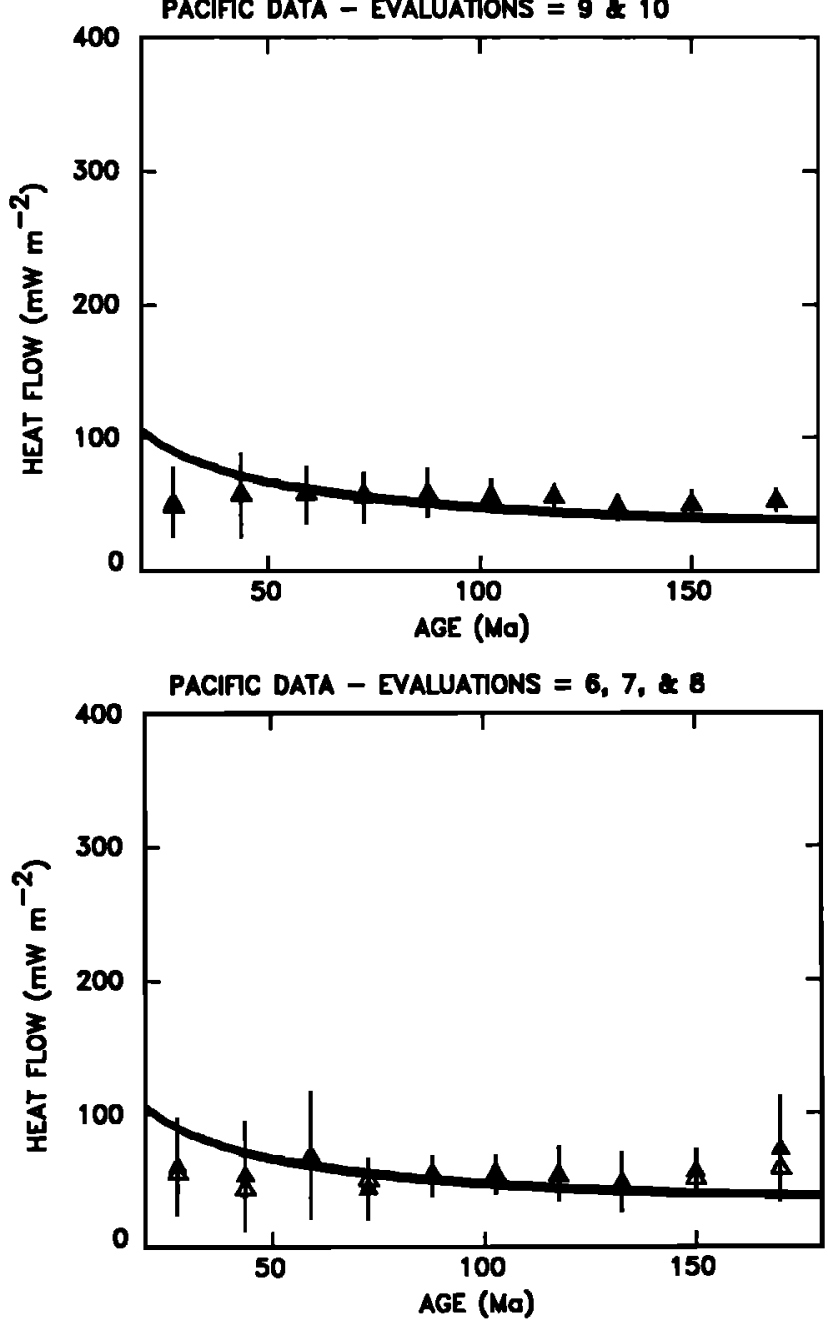

Fig. 3b. Means (solid triangle), medians (open triangle), and standard deviations (vertical line) for the heat flow data in Figure $3 a$. When the median and mean values are comparable, the median symbols are sometimes not visible. The thick solid lines show the heat flow expected for a $125-\mathrm{km}$-thick plate and a $1350^{\circ} \mathrm{C}$ basal temperature. The standard deviations for the poorest category (lower left) generally exceed those for the others.

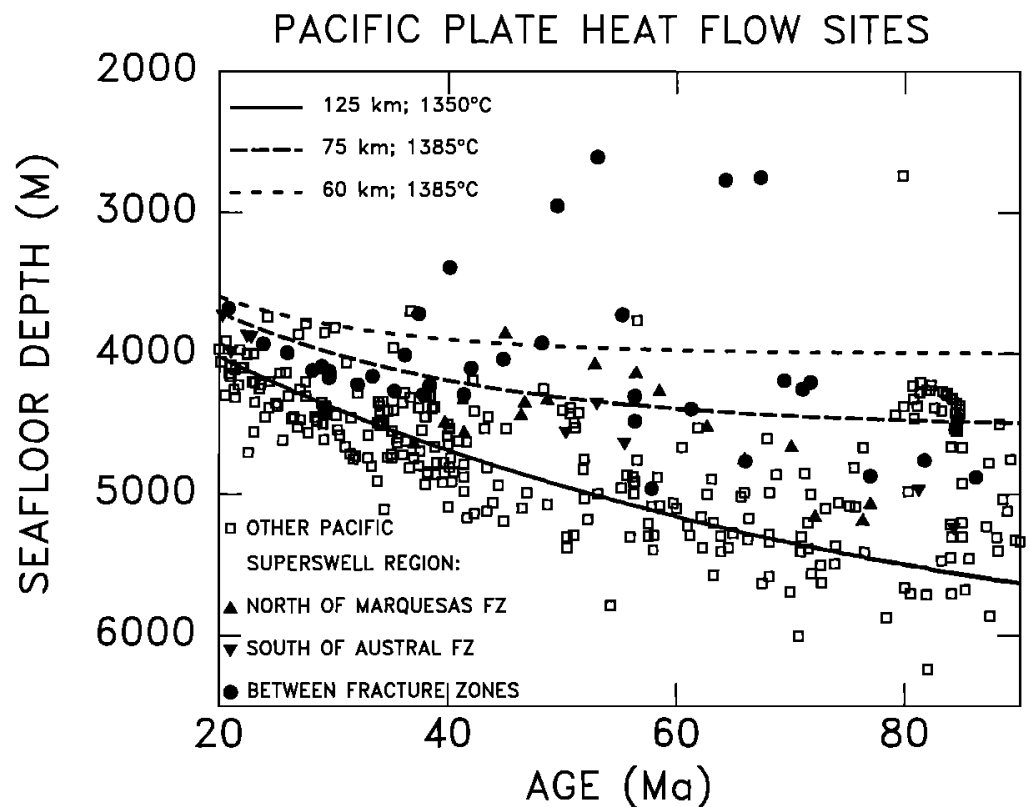

Fig. 4. Seafloor depth and lithospheric age for the Pacific plate heat flow sites. The depths of the heat flow sites in superswell region are better fit by a model with a $75-\mathrm{km}$-hhick plate, whereas the typically assumed $125-\mathrm{km}$-thick plate better fits the depths of the other Pacific plate sites. 


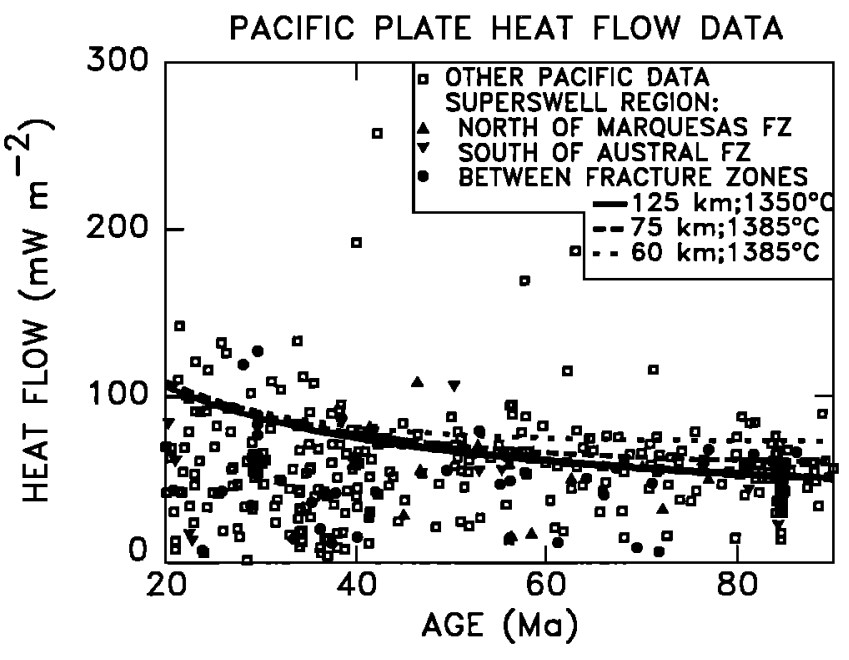

PACIFIC PLATE HEAT FLOW STATISTICS

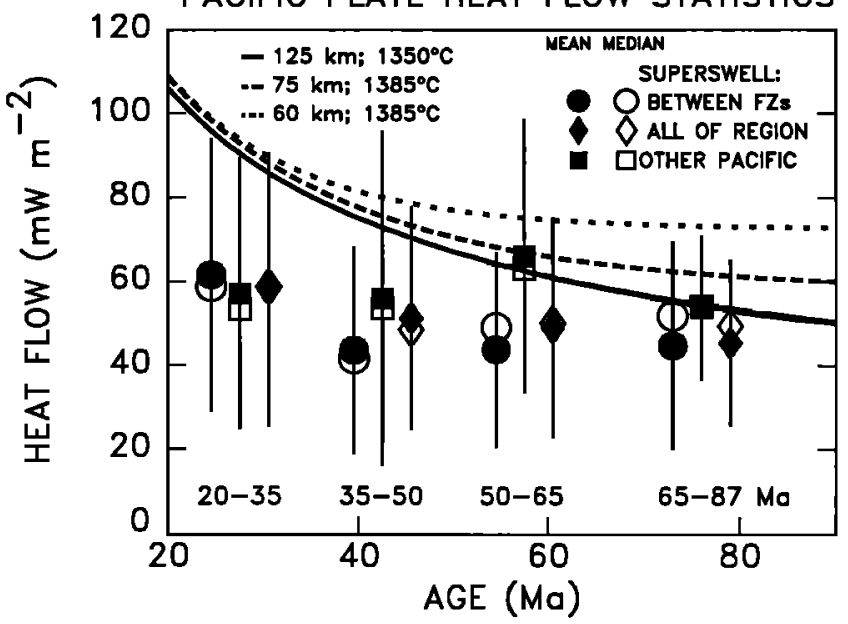

Fig. 5. (top) Heat flow and lithospheric age for sites in the superswell region and elsewhere on the Pacific plate. The heat flow values for the superswell are not generally greater than other Pacific plate values. The heat flow values between the Marquesas and Austral fracture zones, the area of greatest depth anomalies, are not on average greater than those for the whole superswell region. (bottom) Means, medians, and standard deviations of heat flow values in the superswell region, the area between the Marquesas and Austral fracture zones, and elsewhere on the Pacific plate. Given the large standard deviations, the superswell heat flow dala suggest that lithospheric thicknesses exceeds $60 \mathrm{~km}$, but cannot discriminate between greater thicknesses.

transfer occurs by water circulation. For ages greater than about $100 \mathrm{Ma}$ the observed heat flow exceeds that predicted. We discuss this further in the section on the Darwin Rise.

\section{SUPERSWELL REGION}

The heat flow sites provide good regional coverage of the superswell and, for comparison, the region surrounding it (Figure 2). Of the 76 heat flow data, 68 have good (6-10) evaluations and are used in the analysis. The data are divided into three groups: 17 from north of the Marquesas fracture zone, 9 from south of the Austral fracture zone, and 42 between these fracture zones, the region presumably most affected by the formation of the superswell.

The heat flow sites sample the region of shallower bathymetry described by McNutt and Fisher [1987]. Figure 4 shows that the superswell heat flow sites are consistently shallower

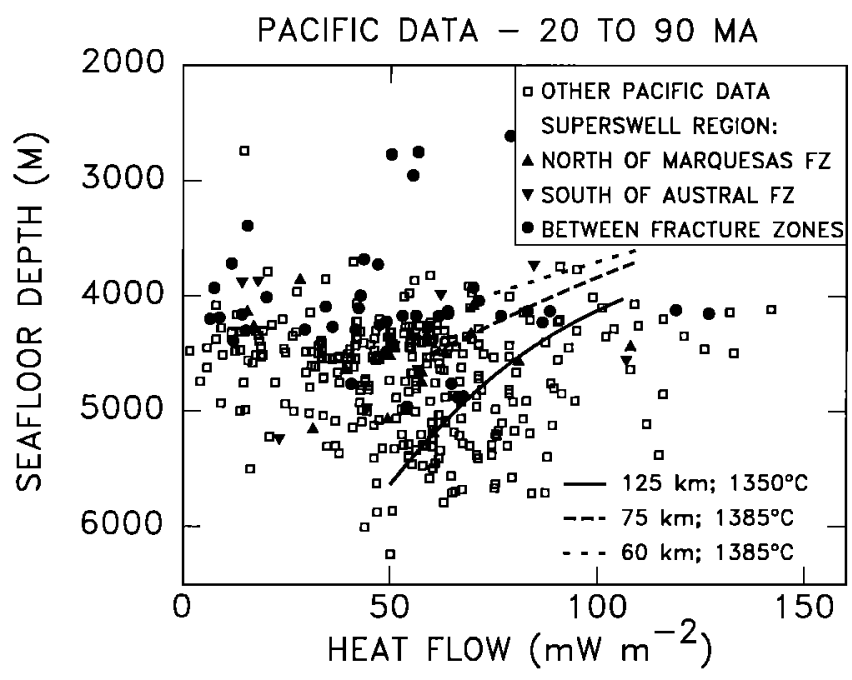

Fig. 6. Seafloor depths and heat flow for the heat flow sites. There does not appear to be any obvious relationship between depth and heat flow for sites either on the superswell or elsewhere on the Pacific plate. The solid and dashed lines, none of which fit the data well, represent the expected relationship between heat flow and depth for models with different plate thicknesses and basal temperatures for lithospheric ages between 20 and $90 \mathrm{Ma}$. Five Pacific values are excluded, one at a depth less than $1000 \mathrm{~m}$ and four with heat flow values greater than $150 \mathrm{~mW} \mathrm{~m}^{-2}$.

than the sites with the same age elsewhere on the Pacific plate. As can be expected, the depths for the superswell siles are better fit by a model with a $75 \mathrm{~km}$ plate, whereas a $125-\mathrm{km}$ plate better fits the depth of the other Pacific plate heat flow sites.

Figure 5 shows the heat flow data versus age for the superswell region and elsewhere on the Pacific plate. The means, medians and standard deviations are shown in Figure 5 (bottom). In this plot, the data are divided into four age bins, each containing an approximately equal number of data for the superswell. Several important points emerge:

1. The heat flow values for the superswell are not significantly higher than for the corresponding ages elsewhere on the Pacific plate.

2. The data show no significant difference between the heat flow for the area of greatest depth anomalies (bctween the Marquesas and Austral fracture zones) compared with the entire superswell region.

3. The heat flow data neither require nor exclude the possibility of a thinner thermal lithosphere: 125 - and $75-\mathrm{km}$-thick plates are admissible for both the superswell and the remainder of the Pacific.

4. The superswell heat flow data exclude the possibility of a plate thinner than $60 \mathrm{~km}$, which would also produce depth anomalies greater than those observed (Figure 4).

Since the superswell was defined by shallow seafloor, we tested whether the heat flow values correlate with depth for the superswell region and the other Pacific sites with similar lithospheric age. No obvious relationship between depth and heat flow appears for either data set (Figure 6). We did not correct for sediment loading, but given the large scatter in the data, such a small correction (probably less than $100 \mathrm{~m}$ for the superswell and generally less than $300 \mathrm{~m}$ for the other Pacific values) would have little effect. Comparison with the trends predicted by plate models for $20-$ to $90-\mathrm{Ma}$ lithosphere shows that the sites with shallower depths do not exhibit the higher 


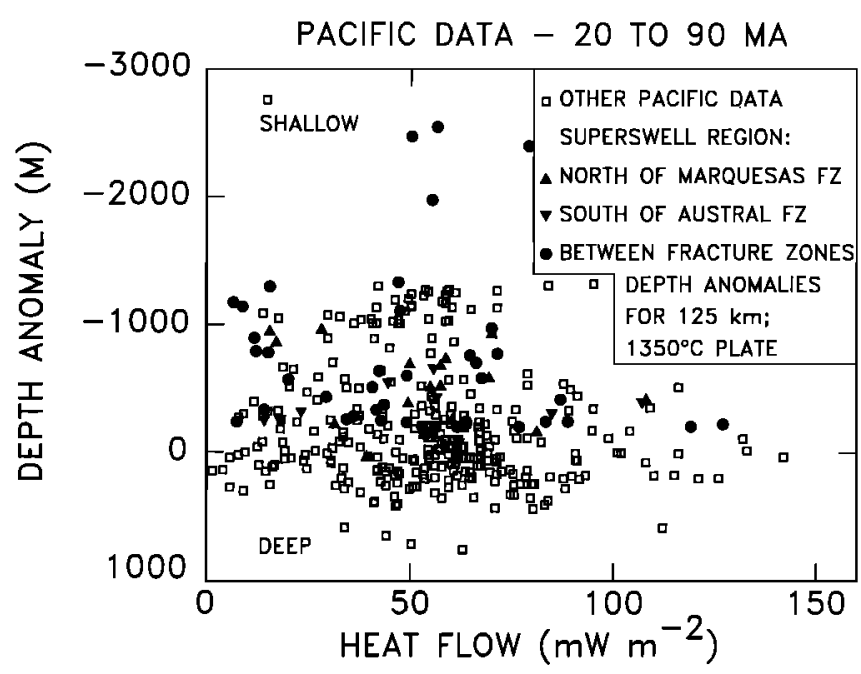

Fig. 7. Depth anomalies with respect to a $125-\mathrm{km}$-thick plate with $1350^{\circ} \mathrm{C}$ basal temperature versus heat flow for the heat flow sites in the superswell region and elsewhere in the Pacific (Figure 6). Although the superswell is defined by shallow bathymetry, there is no obvious correlation between the depth anomaly and the heat flow values.

heat flow expected if the elevation were controlled by lithospheric temperatures. Comparison of the heat flow values to the depth anomalies relative to those for a $125-\mathrm{km}$ plate (Figure 7) also shows no obvious correlation for either the superswell or the rest of the Pacific. Moreover, the depth anomalies show no correlation with the heat flow anomalies relative to a $125 \mathrm{~km}$ thick plate (not plotted).

\section{Possible Biases Due to Water Circulation}

One possible explanation for the absence of measured high heat flow is the removal of heat due to water circulation in the poorly sedimented superswell region. Throughout most of the superswell region there is a very thin sedimentary cover, less than $100 \mathrm{~m}$ (0.1 s two-way travel time) [Ludwig and Houtz, 1979]. The sedimentary environment category for 38 of the 76 heat flow measurements are known. Two are in category A, 16 are in $B, 12$ are in C, and 8 are in D. Nine of the sixteen B sites are from the detailed survey at $18^{\circ} \mathrm{S}, 130^{\circ} \mathrm{W}$. Hence most of the superswell heat flow measurements are at sites with $C$ and $D$ environments.

To test if the observed superswell heat flux is abnormal, we compared the data from superswell sites with $C$ and D environments, and those for which we had insufficient data to determine the environment, with 39 measurements at $C$ and D sites elsewhere in the Pacific and 45 from such sites in the Atlantic [Herman, 1987] (Figure 8). Again, we find no significant differences between the average values for the superswell and the other oceans, nor between the Marquesas and Austral fracture zones and the areas directly to the north and south. In some cases, the superswell data are somewhat lower than the other regions.

We have other reasons for doubting that water circulation seriously biases our results. At sites where heat flow was determined from three or more temperature measurements with depth, the possible effect of water flow and the flow velocity can often be measured. Vertical water flow results in temperature gradients that are nonlinear [Bredehoeft and Papadopulos, 1965]. Of the 24 sites at which three or more temperatures were measured, only one (C11-116) shows a nonlinear gradient. Given this observation and the comparison with the $C$ and $D$

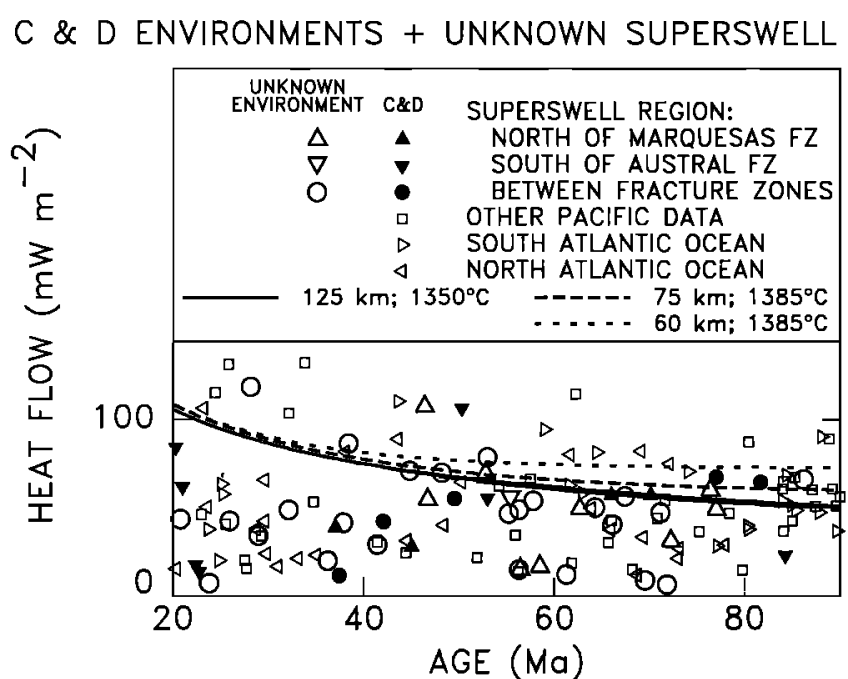

Fig. 8. Heat flow at superswell sites with $C$ and $D$ environments elsewhere in the Pacific and the Atlantic versus age. Superswcll sites with insufficient data to determine the sedimentary environments are also included, since most of the sites with determined environments are in the $C$ or $D$ categories. Heat flow values for the superswell region are not high compared to those at the other sites.

sites elsewhere, we consider it unlikely that the absence of a large observed heat flow anomaly for the superswell region results from a bias due to water circulation near the sea floor.

\section{DARWIN RISE}

We also examined heat flow data for the Darwin Rise, the region of rough relief in the western Pacific that $M c N u t t$ and Fisher [1987] suggested was a fossil superswell in the Cretaceous on the basis of the many characteristics similar to those of the South Pacific superswell. Halumen and Von Herzen [1973] noted that the heat flow across some of this region was relatively constant and proposed that any additional heat flow associated with the Darwin Rise had been dissipated. We analyzed additional heat flow data gathered since that study and used subsequent studies that determined lithospheric ages for the region.

The precise boundaries of the Darwin Rise region are difficult to define, since a large area of the present westem Pacific plate was affected by a diffuse pattern of volcanic activity during the Cretaceous [Schlanger et al., 1981]. We chose the study area to include a region surrounding the continuation of hot spot tracks from the present-day superswell, an area roughly south and east of Hawaii and north of the Samoan hot spot track. Heat flow data are available at 124 sites in the region (Figure 9) of which 114 have evaluations between 6 and 10 (categories A and B). The depths for the Pacific plate heat flow sites (Figure 10) are about $700 \mathrm{~m}$ deeper than might expected for a $75-\mathrm{km}$-thick plate. They are, however, shallower than might be expected for a $125-\mathrm{km}$ - thick plate. McNutt et al. [1990] suggest that the depth anomaly is consistent with their proposed tectonic history of the region, including the termination of superswell conditions about 70-80 Ma.

The heat flow values for the Darwin Rise, with lithospheric ages of about 95-173 Ma, are similar to the rest of the Pacific plate (Figure 11, top). In each of the four age bins (Figure 11, bottom) the difference of the means between the Darwin Rise data and that elsewhere in the Pacific is less than $10 \mathrm{~mW} \mathrm{~m}^{-2}$ and the maximum difference in median value is $7 \mathrm{~mW} \mathrm{~m}^{-2}$.

A potential difficulty with comparison of the Darwin Rise data with data of comparable age elsewhere in the Pacific is that a large region of the Pacific plate outside of the Darwin Rise 
was also affected by Mesozoic volcanism. We thus also compared the Darwin Rise heat flow data with data for similar age lithosphere in the Atlantic. For this comparison, we used only the best data, stations with known A sedimentary environments. Again, the Darwin Rise values are no higher than for the other areas (Figure 12).

We regard the observation that the Darwin Rise heat flow does not exceed that for lithosphere of comparable age elsewhere in the Pacific and Atlantic as significant and as illustrating that whatever thermal changes may have been associated with the formation of the Darwin Rise are no longer present. This would be consistent with the idea of a fossil superswell. We do not, however, ascribe great significance to the fact that the Darwin Rise data heat flow values exceed those predicted for a 125-km-thick plate. This discrepancy occurs elsewhere for ages exceeding about $100 \mathrm{Ma}$, an effect previously noted whose cause remains obscure [Sclater et al., 1980; Davis et al., 1984; Detrick et al., 1986; Courtney and White, 1986; Louden et al., 1987; Davis, 1989; Lister et al., 1990]. An additional complexity for the Darwin Rise, and quite possibly elsewhere, is that volcanic edifices formed far from the ridge crests contribute

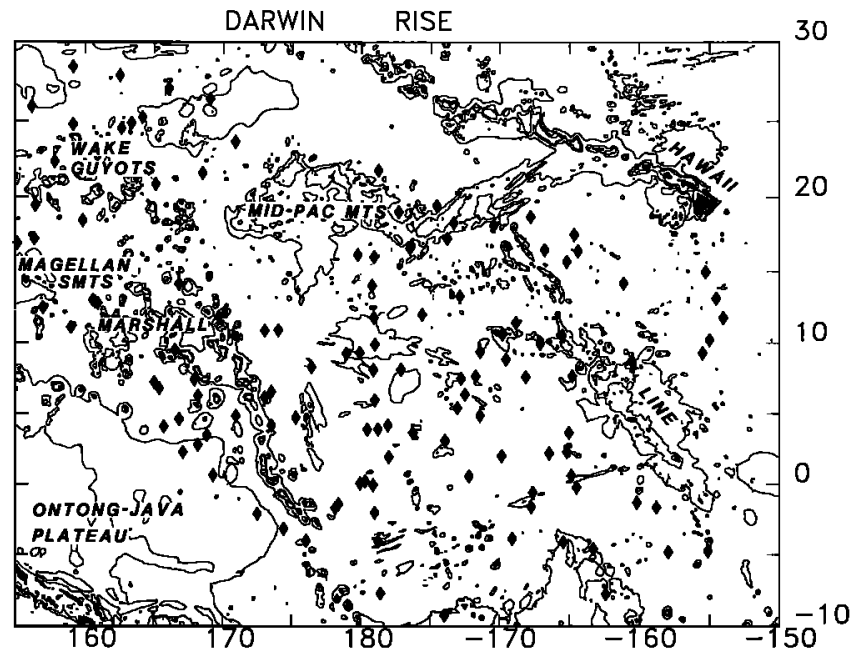

Fig. 9. Darwin Rise bathymetry (1500-m contours) and heat flow locations (solid diamonds).

PACIFIC PLATE HEAT FLOW SITES

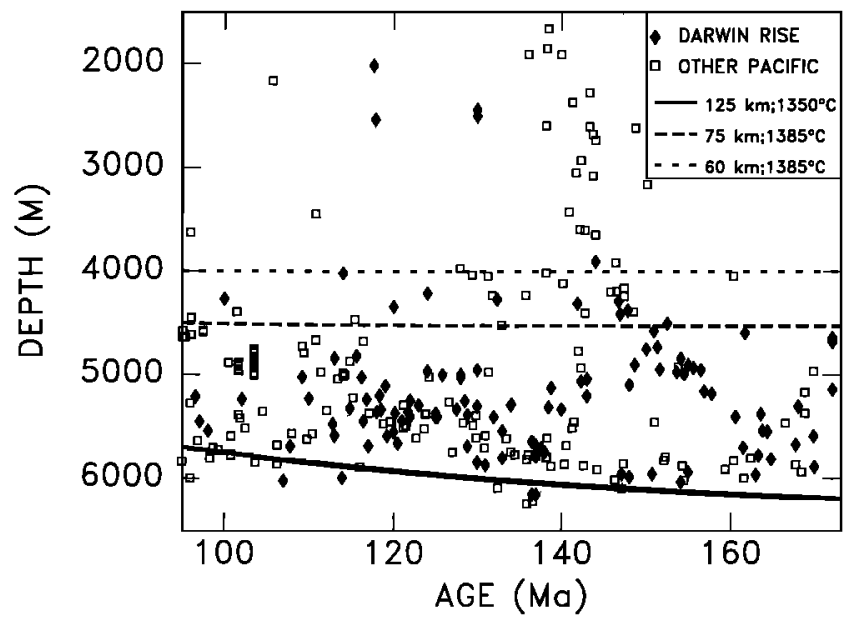

Fig. 10. Depth versus age for the Darwin Rise and other Pacific plate heat flow sites. The depths for both areas are shallower than might be expected for their age, assuming a $125-\mathbf{k m}$-thick plate, but deeper than might be expected for a $75-\mathrm{km}$-thick plate.
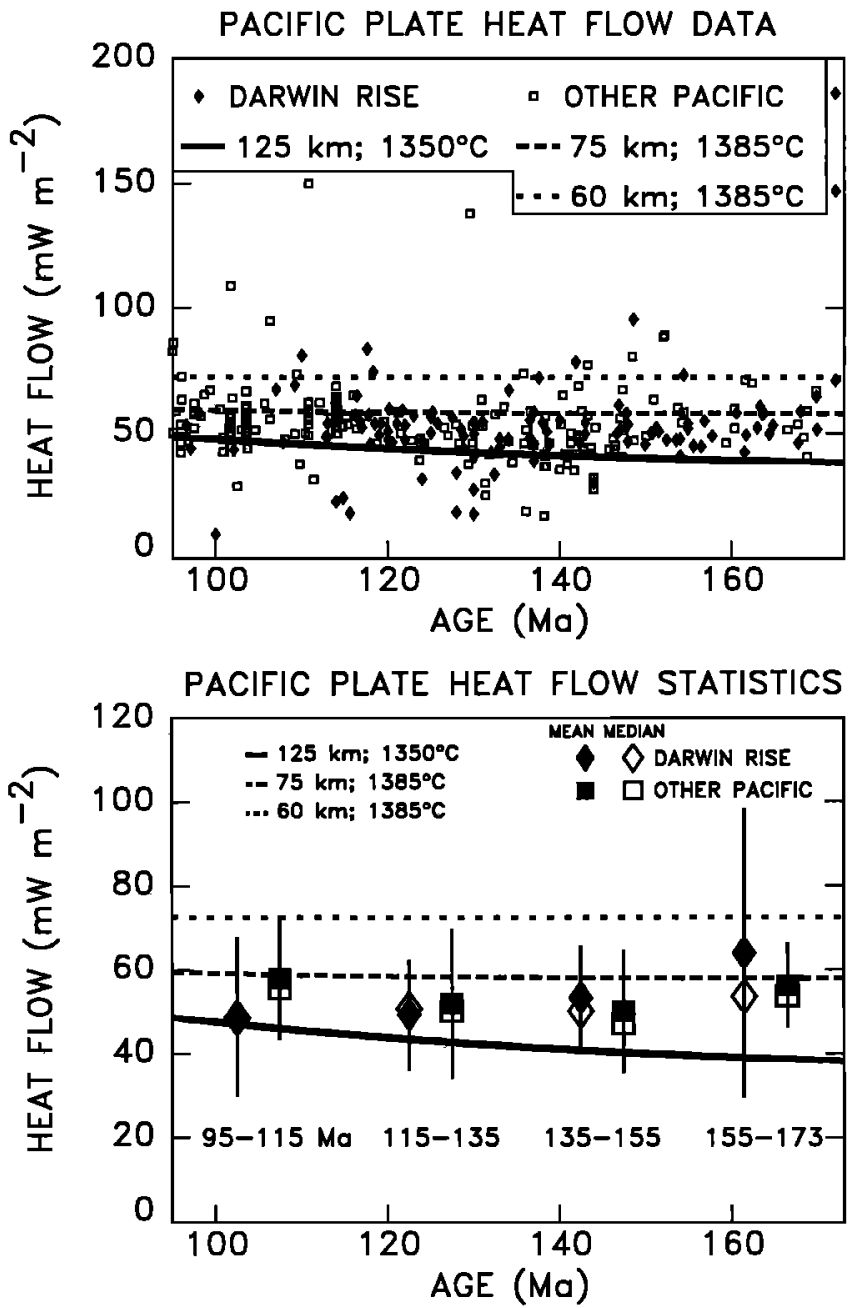

Fig. 11. (top) Heat flow versus age for sites on the Darwin Rise and Pacific plate. On average the values from the Darwin Rise are similar to those for the rest of the Pacific plate. The average heat flow exceeds that expected for a $125-\mathrm{km}$-thick plate model but is typical of data for similar ages on other plates. (bottom) Means, medians, and standard deviations for the Darwin Rise and other Pacific plate heat flow measurements. The relatively large difference between the median and mean values for the $155-173 \mathrm{Ma}$ age bin results from the high heat flow of stations V6333-25 and V6333-27 (Table 2).

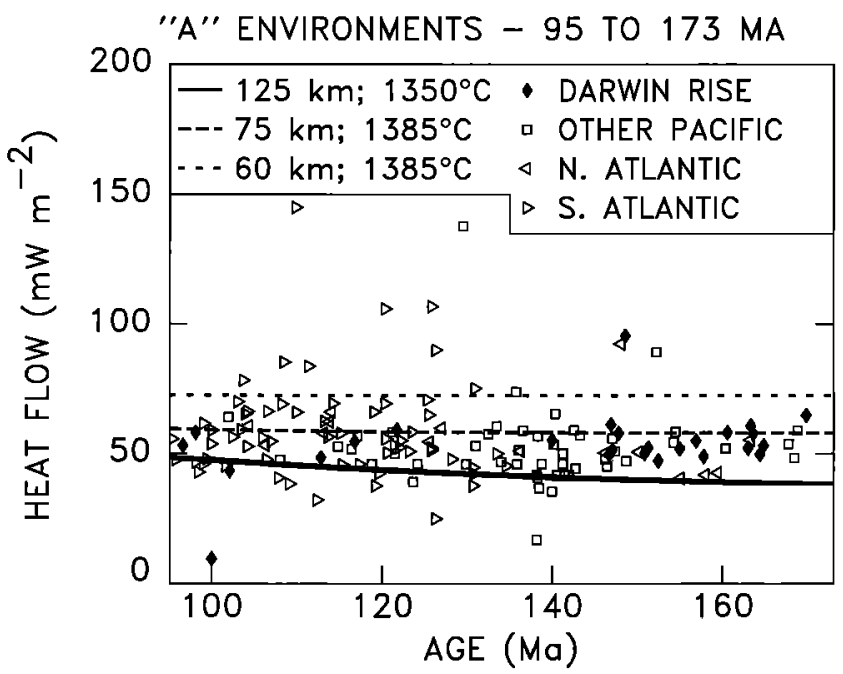

Fig. 12. Heat flow versus age for sites with known $A$ environments on Darwin Rise, elsewhere on the Pacific plate, and the Atlantic. The Darwin Rise heat flow is not anomalously high compared with the other regions. 
significantly to the topography, further obscuring the relation between heat flow and depth.

\section{THeRMal Models}

We next consider the implications of the heat flow data and other observations for the thermal structure and evolution of the superswell region. For this purpose, we investigated which thermal structures might reconcile two basic constraints. First, the heat flux for the superswell and the Darwin Rise is no higher than for lithosphere of comparable age elsewhere in the Pacific. Second, the effective elastic thickness of the lithosphere in the superswell region is significantly less than that for lithosphere of comparable age elsewhere.

\section{Effective Elastic Thickness}

The effective elastic thickness $T_{e}$ of the lithosphere, as inferred from the deflections due to loads such as seamounts, generally increases with the square root of the age of the lithosphere at the time of loading [Watts et al., 1980]. This effect is thought to reflect the decrease in lithospheric strength with depth due to increasing temperature, such that the effective elastic thickness as a function of age can be approximated as controlled by the depth to an isotherm [Bodine et al., 1981]. A global compilation derived using the Seasat gravity and SYNBAPS bathymetric data bases shows that the effective elastic thicknesses beneath 18 islands and seamounts in the superswell region are anomalously low for their age of loading (Figure 13) compared with 20 other Pacific sites [Calmant 1987; Calmant and Cazenave, 1987], 11 Atlantic, and 6 Indian Ocean sites [Calmant et al., 1990]. The calculation of effective elastic thickness from an observed deflection depends, of course, on a variety of model parameters. McNutt and Judge [1990] also note, using somewhat different values, that the effective elastic thicknesses in the superswell are significantly lower than for hot spots elsewhere. For our modeling, we use the larger Calmant et al. [1990] data set.

The low effective elastic thicknesses have been used to support the suggestion of higher than normal temperatures associated with either multiple rejuvenation due to hot spots or the more recently proposed superswell model for the South Pacific and the Darwin Rise. Menard and McNutt [1982] suggested that multiple rejuvenation events due to the passage of the young lithosphere over numerous hot spots might explain the shallow depths, high density of volcanoes, and low effective elastic thicknesses. Calmant and Cazenave [1987] suggested that multiple rejuvenation events might explain their data. McNutt and Fisher [1987], however, proposed that multiple rejuvenation had to affect a very large region and would presumably not give rise to a systematic variation in the depth anomalies with age. They thus hypothesized that the superswell mantle anomaly is a transient feature, whereas the hot spots are longer duration phenomena which give rise to surface volcanism more easily when the lithosphere is thinned under superswell conditions.

We explored a range of steady state and time dependent (reheating) thermal models to see whether the thin effective elastic thicknesses can be reconciled with the absence of a heat flow anomaly. Our starting point was the constraint that the effective elastic thicknesses inferred for sites outside the superswell correspond to depths between the $300^{\circ}$ and $600^{\circ} \mathrm{C}$ isotherms predicted for a $125-\mathrm{km}$-thick plate thermal model. We used the simple assumption that if the low effective elastic thicknesses for the superswell reflect thermal structure, these
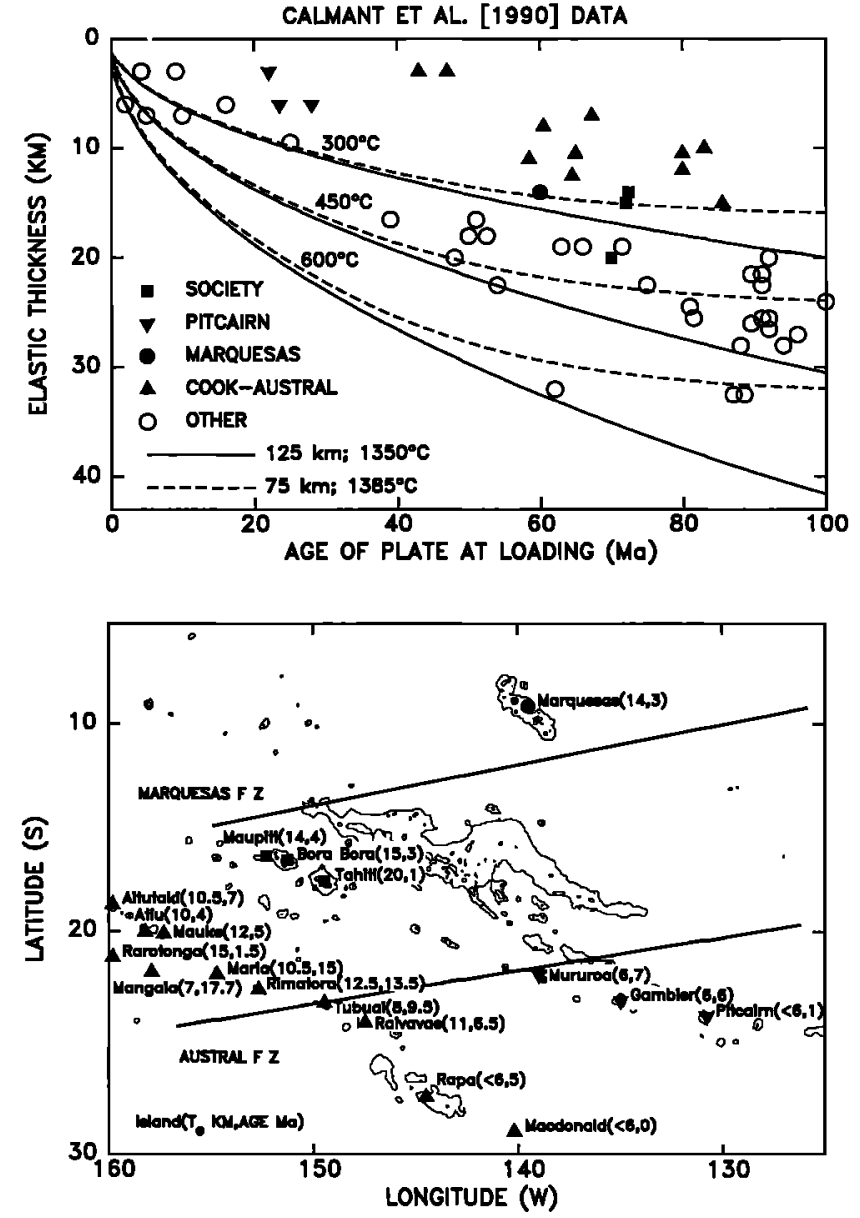

Fig. 13. (top) Effective elastic thickness $\left(T_{e}\right)$ versus age of the plate at loading, and isotherms predicted for $125-\mathrm{km}$ and $75-\mathbf{k m}$-thick plates. A 75-km-thick plate does not have sufficiently higher temperatures at upper lithospheric depths compared with the 125-km-thick plate to account for the lower $T_{e}$. (bottom) Location of $T$, measurements in the superswell region. The different seamount chains are indicated by the symbols from the upper panel. The numbers in parentheses are the $T_{e}$ in $\mathrm{km}$ and age of volcanism in Ma [Calmant et al., 1990]. The 1000- and 3500-m contours are shown.

thicknesses should be between the elevated $300^{\circ}$ and $600^{\circ} \mathrm{C}$ isotherms. We examined models that achieve this weakening to see whether the predicted heat flow exceeded that observed.

\section{Steady State Models}

The simplest model that can give rise to different elevations and surface heat flow is one in which the plate evolves with different thickness and/or basal temperatures than those normally assumed $\left(125 \mathrm{~km} ;-1350^{\circ} \mathrm{C}\right)$. For example, thinner plate thicknesses and higher basal temperatures will result in higher surface heat flow and shallower bathymetry. Such a mechanism for the observed heat flow anomalies in the central Indian Ocean was considered and then rejected given the lack of regional depth anomalies [Stein and Weissel, 1990].

We compared the predicted depths of the isotherms (Figure 13 , top) for a $125-\mathrm{km}$-thick plate and the $75-\mathrm{km}$-thick plate proposed by McNutt and Fisher [1987]. The depths to the isotherms in the upper lithosphere for a $75-\mathrm{km}$-thick plate are only slightly shallower than for "normal" lithosphere with the age range at the time of loading for the South Pacific superswell. This result is not unexpected, as conductive heat transfer 
requires a finite time for the location of the bottom boundary to significantly affect the cooling process. Hence a $75-\mathrm{km}$-thick plate alone is insufficient to explain the low effective elastic thickness data. A $40-\mathrm{km}$-thick plate with a $1350^{\circ} \mathrm{C}$ basal temperature or a $75-\mathrm{km}$-thick plate with a $2000^{\circ} \mathrm{C}$ basal temperature can raise the isotherms sufficiently to fit the effective elastic thickness data (Figure 14, top). Such models, however, predict heat flow substantially greater than observed (Figure 14, middle) and seafloor depths much shallower than observed (Figure 14, bottom). In addition, such lithospheric thicknesses or basal temperatures seem geologically unreasonable.
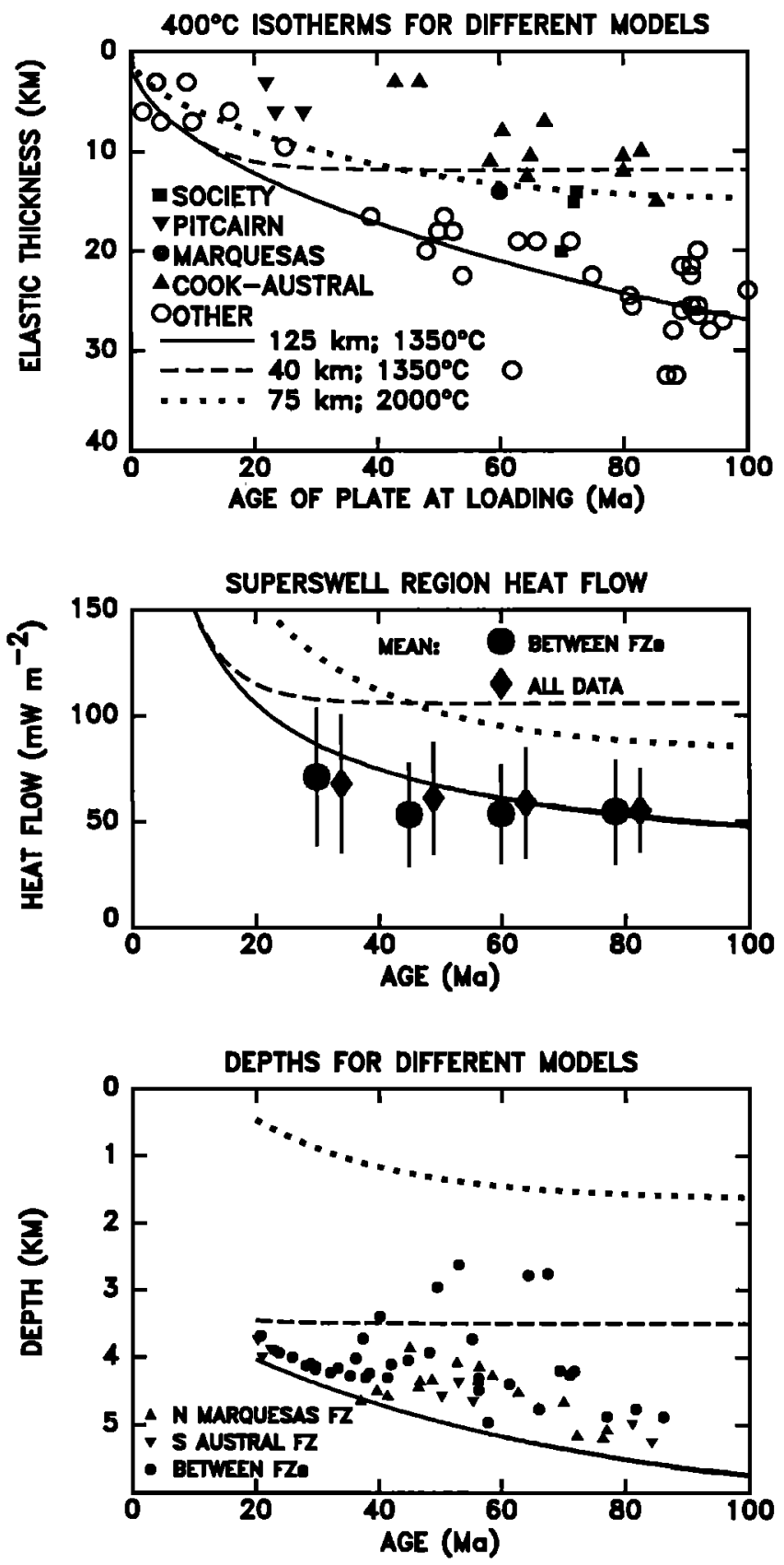

Fig. 14. (top) Measured effective elastic thicknesses and depths to the $400^{\circ} \mathrm{C}$ isotherm for three different models of basal temperature and plate thicknesses. (middle) Heat flow expected for the different models. (bottom) Depths and ages of the heat flow measurement sites in the superswell and those expected for the different models. Models having sufficiently high upper lithospheric temperatures to fit the $T_{e}$ data have too high heat flow and elevations compared with the data.

\section{Time Dependent Models}

An alternative class of models are those in which the thermal structure of the lithosphere is perturbed at some time and then evolves. The effect on surface heat flow from changes in the temperature with depth depends on the depth of the perturbation and the time since its initiation [Carslaw and Jaeger, 1959].

Unfortunately, it is difficult to date the formation of the superswell. We can estimate a minimum age provided that the thin effective elastic thicknesses beneath seamounts in the superswell area imply that these loads were emplaced by hot spots subsequent to the formation of the superswell. Since the oldest seamount in the superswell with thin $T_{e}$, Mangaia, in the Cook-Austral Islands, is dated at 17.7 Ma [Calmant, 1987], anomalous conditions must predate its formation. Even under this assumption, of course, the superswell might be significantly older.

For a hot spot reheating model, in which the bottom portion of the lithosphere is reset to asthenospheric temperatures [Crough, 1978; Detrick and Crough, 1978], the depth to reheating determines the anomalous bathymetry and heat flow with time. The shallower the reheating depth, the greater the depth anomaly, the sooner the maximum surface heat flow anomaly occurs, and the greater its magnitude. The depth for reheating is estimated largely from bathymetry. Crough [1978] proposed that hot spots reset the temperature structure to that of a $25-\mathrm{Ma}$ lithosphere, and McNutt [1987] proposed that reheating occurred up to the initial location of the $900^{\circ} \mathrm{C}$ isotherm.

To approximate the conditions for the superswell, we calculated the temperature structure and heat flow predicted by the half-space reheating model of Von Herzen et al. [1982]. Reheating to the depth of the $900^{\circ} \mathrm{C}$ isotherm for lithosphere in the range of ages for the superswell results in very little shallowing of the $400^{\circ} \mathrm{C}$ isotherm. For example, Figure 15 shows unperturbed (solid line) and perturbed (dashed line) isotherms and surface heat flow with time expected for the reheating of $45-\mathrm{Ma}$ lithosphere to the depth of the $900^{\circ} \mathrm{C}$ isotherm, $45 \mathrm{~km}$. Although the heat flow anomaly predicted is small enough to not violate our heat flow data constraint, the increase in temperature in the upper lithosphere is insufficient to explain the effective elastic thickness data. Much shallower reheating depths are required to elevate the temperatures sufficiently to fit the $T_{e}$ data. For example, 45-Ma lithosphere must be thinned to 15 $\mathrm{km}$ depth (Figure 16) to place the largest effective elastic thicknesses in the expected temperature range. Such thinning would give rise to predicted heat flow much larger than observed. Moreover, such a shallow reheating depth would be substantially shallower than that previously proposed for other hot spots. The same difficulties occur for reheating at the other lithospheric ages in the superswell.

\section{DiscUSSION}

The heat flow data and our modeling demonstrate the difficulties in interpreting the low effective elastic thicknesses for the South Pacific and the fossil Darwin Rise superswells in terms of thermal weakening due to high upper lithospheric temperatures. Since the $T_{e}$ values are about half those for lithosphere outside the superswells, the isotherms should be at about half the normal depths, and the surface heat flow should be about twice that expected. Since such high heat flow is not observed, we feel that regional reheating sufficient to explain the effective elastic thickness data is unlikely.

In addition, a regional reheating model has other difficulties. The sites in the region of maximum depth anomalies (between 
ELASTIC THICKNESS AND THERMAL STRUCTURE
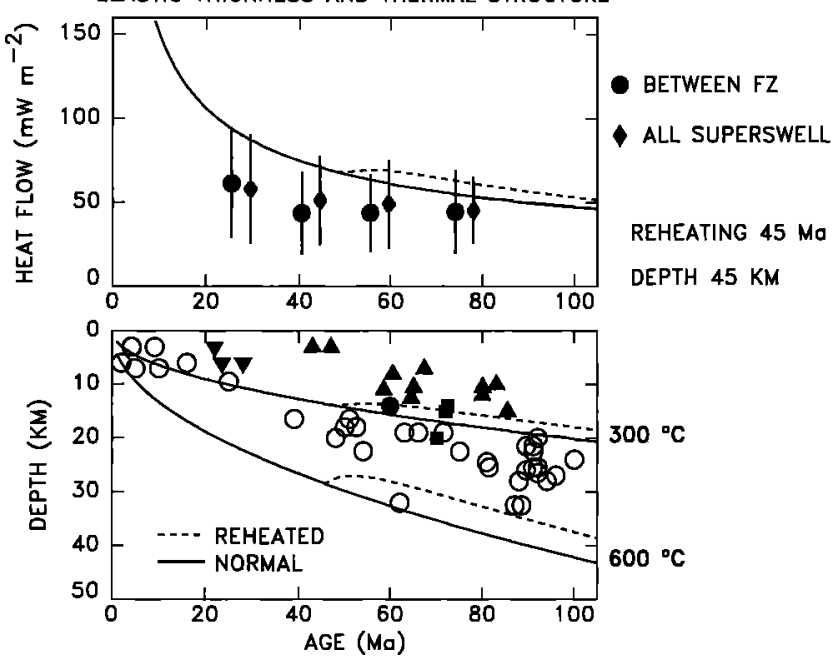

Fig. 15. Model for reheating 45-Ma lithosphere to a depth of $45 \mathrm{~km}$. Although the calculated heat flow anomaly (top) is plausible given the observed heat flow, such a model does not give sufficient reheating of the upper lithosphere (bottom) to account for the low $T_{e}$. In the bottom panel, the seamount chains are indicated by symbols used in Figure 13.
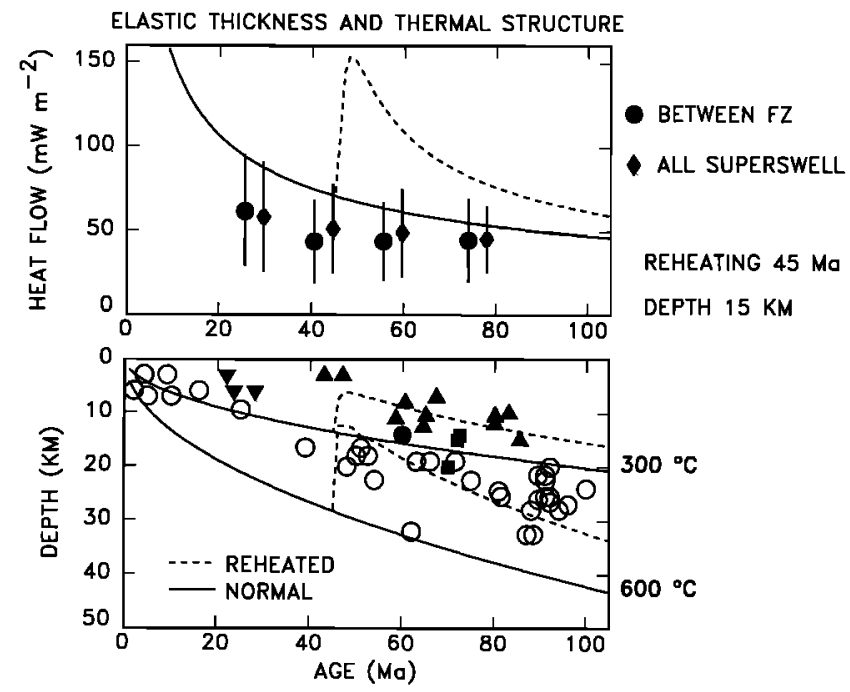

Fig. 16. Model for reheating 45-Ma lithosphere to a depth of $15 \mathrm{~km}$. Although this model produces sufficient reheating in the upper lithosphere to account for the largest $T_{e}$ of the superswell sites, it predicts a heat flow anomaly significantly larger than that observed. In the bottom panel, the seamount chains are indicated by symbols used in Figure 13.

the Marquesas and Austral fracture zones), which should be the most affected by the superswell, do not have the lowest effective elastic thicknesses. The Society Islands (near the center of the superswell) and the Marquesas have about the same $T_{e}$ (Figure 13), although the latter is not in the superswell since the only depth anomalies north of the Marquesas fracture zone are those associated with this volcanic chain [McNutt and Fisher, 1987]. Of the three measurements in the Society Islands, Tahiti has the highest $T_{e}$ for all the 18 superswell region sites, but this large value may result from the site not being in equilibrium thickness yet [Calmant and Cazenave, 1986]. However, this does not explain the relatively high values for Bora-Bora and Maupiti. Conversely, the five sites with the thinest effective elastic thicknesses (Pitcaim, Gambier,
Mururoa, MacDonald, and Rapa) are south of the Austral fracture zone and hence outside the region of maximum depth anomalies. Finally, if regional reheating occurred then sites with the larger depth anomalies should be associated with higher heat flow, but this is not observed (Figure 7).

Our observation of a lack of a heat flow anomaly for the superswell and inference that significant shallow regional reheating has not occurred is consistent with new results for the Hawaiian hot spot chain. Von Herzen et al. [1989] suggest, from detailed heat flow data and comparison with other old lithosphere, that little reheating has occurred. They suggest that their data are compatible with at most about $100^{\circ}-200^{\circ} \mathrm{C}$ increase at the base of the plate and hence that a model assuming a significant dynamic component to explain the elevation of hot spots is required. In addition, recent results show that surface wave velocities along the Hawaiian-Emperor chain from Midway to Oahu are similar to those expected for unperturbed $100-\mathrm{Ma}$ lithosphere, and significantly faster than those expected if the Hawaiian swell originates primarily by large-scale reheating [Woods et al., 1991].

What altematives are there to a regional reheating model? We can see three possibilities that might reconcile the lack of high heat flow and the thin effective elastic thicknesses.

First, the heat flow and $T_{e}$ observations could be consistent if the high temperatures occur only below the seamounts rather than throughout the region. Unfortunately, since the heat flow measurements were not taken to address this issue, only a few are within $200 \mathrm{~km}$ of the seamounts beneath which $T_{e}$ has been determined (Figure 2). The few measurements close to these seamounts do not show dramatically high heat flow. The heat flow values for the Marquesas (RIS 145, 146, and 147) and for the Society (RIS 136, 137, and 138) sites are not anomalous relative to the means and standard deviations for the age group. For the Austral-Cook chain, C13-132 and 133 and CAP-31B are a few $\mathrm{mW} \mathrm{m}^{-2}$ greater than the mean plus 1 standard deviation, but less than would be expected if the plate were thermally weakened. Nonetheless, given the limited data and their location, we cannot exclude local reheating to very shallow depths.

A natural question posed by the possibility of local reheating is why such a phenomenon would occur for the superswell hot spots but not others, since the effective elastic thicknesses observed near other hot spots (Figure 13) are significantly greater. The hotspots of the superswell region are not thought to be exceptional ones; each of the four hot spots (Pitcaim, Marquesas, MacDonald, and Tahiti) has about 3/8 the buoyancy flux of Hawaii [Sleep, 1990]. Similarly, McNutt and Judge [1990] proposed that the concentration of hot spots in the superswell occurs because the thinned lithosphere is easily penetrated by numerous, but relatively weak, thermal plumes that might be incapable of producing midplate volcanoes on normal lithosphere. Hence these hot spots often produce volcanoes only intermittently as a result of preexisting weaknesses in a plate and/or the thinner plate associated with the formation of the superswell [McNutt and Fisher, 1987; McNutt et al., 1989]. Inferred geotherms from analyses of mantle xenoliths would be useful, but few data are available for the superswell region. A study of ultramafic xenoliths, consisting of mainly spinel lherzolites, near Papeete (Tahiti), suggests temperatures of $\sim 1100^{\circ} \mathrm{C}$ at pressures less than about 15-20 kbar [Tracy, 1980] (about $45-60 \mathrm{~km}$ ) and thus suggests that very shallow reheating is not occurring.

Second, the low effective elastic thicknesses might result from mechanical rather than thermal weakening of the lithosphere. The presence of a significant amount of volatiles, such 
as water, might result in a weaker rheology. Alternatively, weakening might be due to successive intrusion events resulting in cracking, flexing and reheating the lithosphere. This hypothesis has the same difficulty as that of local reheating under the seamounts, in that it is unclear why the superswell hot spots should show this effect whereas others do not. Perhaps the explanation lies in the complex tectonic history of the region. The Cook-Austral island chain has many anomalous features and is less linear and more segmented than the Hawaiian chain [Okal and Batiza, 1987]. Turner and Jarrard [1982] proposed that the Cook-Austral chain violated the age-distance relationship predicted for a single hot spot and suggested that a hot spot model for the chain would require three hot spots. Also, an area through part of the chain may have been affected by hot spot activity sometimes between the Late Cretaceous and Eocene [Diament and Baudry, 1987]. The Pitcaim chain was emplaced on lithosphere previously affected by the Easter hot spot (Gordon and Henderson, unpublished manuscript, 1985) and may have a complicated pattern of volcanism due to the complex interaction of the hot spot with fracture zones [O kal and Cazenave, 1985]. Although such effects might explain the unusual low $T_{e}$ for the Cook-Austral and Pitcairn chain, it is more difficult to explain the low values for the Society and Marquesas.

Third, the low effective elastic thicknesses could reflect regional intraplate stresses resulting from plate boundary forces, such as suggested for the Indian Ocean [Cloetingh and Wortel, 1985, 1986; Stein et al., 1989], or from the mantle upwelling associated with the superswell. The flexural response of the lithosphere to an applied load depends on both the mechanical properties and the regional stress [Turcotte and Schubert, 1982]. This possibility is suggested by the presence in the superswell area of widespread seismicity, some of whose epicenters seem unrelated to volcanic events, in contrast to Hawaii where most of the epicenters appear related to active volcanoes [Wysession et al., 1991]. However, a simple line load model assuming elastic behavior [Hetenyi, 1974] suggests that to reduce the effective elastic thicknesses by even $20 \%, \sim 400 \mathrm{MPa}(4 \mathrm{kbar}$ ) of compressional stress would be required. It seems unlikely that intraplate stresses would be so large given that the level of Pacific intraplate seismicity is not as high as that in the Indian Ocean.

\section{CONCLUSIONS}

The absence of a regional heat flow anomaly for the superswell argues against a regional reheating of the upper lithosphere sufficient to explain the thin effective elastic thicknesses as a consequence of thermal weakening. The heat flow and effective elastic thickness data might perhaps be reconciled if beneath seamounts in the superswell area either major shallow reheating or mechanical weakening occurs. Additional data on the effective elastic thicknesses of superswell seamounts and their ages, and additional heat flow data near these seamounts, would be useful for better understanding the dynamics and evolution of the South Pacific superswell and the Darwin Rise.

Acknowledgments. This research was supported by grants 21625-G2 and 19134-AC2-C from the Petroleum Research Fund, administered by the American Chemical Society and ONR N00014-87-K-204. We thank Richard Gordon, David McAdoo, Marcia McNutt, Emile Okal, David Sandwell, Sy Schlanger, Seth Stein, and Mark Woods for useful discussions and advice. We thank Mark Hobart for permission to use the
ARIAD heat flow data. We thank C. R. B. Lister for a preprint of his manuscript. Lamont-Doherty Geological Observatory contribution 4784 .

\section{REFERENCES}

Abbott, D., and M. Fisk, Tectonically controlled origin of three unusual rock suites in the Woodlark Basin, Tectonics, 5, 1145-1160, 1986.

Anderson, R. N., and M. A. Hobart, The relation between heat flow, sediment thickness, and age in the eastem Pacific, J. Geophys. Res., 81, 2968-2989, 1976.

Anderson, R. N., and R. P. Von Herzen, Heat flow on the PacificAntarctic Ridge, Earth Planet. Sci. Lett., 41, 451-460, 1978.

Anderson, R. N., M. A. Hobart, R. P. Von Herzen, and D. J. Fornari, Geophysical surveys on the East Pacific Rise-Galapagos Rise system, Geophys. J. R. Astron. Soc., 54, 141-166, 1978.

Bodine, J. H., M. S. Steckler, and A. B. Watts, Observations of flexure and the rheology of the oceanic lithosphere, J. Geophys. Res., 86, 3695-3707, 1981 .

Boyce, R. E., Electrical resistivity, sound velocity, thermal conductivity, density-porosity and temperature, obtained by laboratory techniques and well logs: Site 462 in the Nauru Basin of the Pacific Ocean, Initial Rep. Deep Sea Drill. Proj., 61, 743-761, 1981.

Bredehoeft, J. D., and I. S. Papadopulos, Rates of vertical groundwater movement estimated from the Earth's themal profile, Water Resour. Res., 2, 325-328, 1965.

Calmant, S., The elastic thickness of the lithosphere in the Pacific Ocean, Earth Planet. Sci. Lett., 85, 277-288, 1987.

Calmant, S., and A. Cazenave, The effective elastic lithosphere under the Cook-Austral and Society Islands, Earth Planet. Sci. Lett., 77, $187-202,1986$.

Calmant, S., and A. Cazenave, Anomalous elastic thickness of the oceanic lithosphere in the south-central Pacific, Nature, 328, 236-238, 1987.

Calmant, S., J. Francheteau, and A. Cazenave, Elastic layer thickening with age of the oceanic lithosphere: A tool for prediction of age of volcanoes or oceanic crust, Geophys. J. Int., 100, 59-67, 1990.

Carslaw, H. S., and J. C. Jaeger, Conduction of Heat in Solids, pp., 510 Oxford University Press, New York, 1959.

Castillo, P., The Dupal anomaly as a trace of the upwelling lower mantle, Nature, 336, 667-670, 1988.

Cloetingh, S., and R. Wortel, Regional stress field of the Indian plate, Geophys. Res., Lett., 12, 77-80, 1985.

Cloetingh, S., and R. Wortel, Stress in the Indo-Australian plate, Tectonophysics, 132, 49-67, 1986.

Cochran, J. R., Variations in subsidence rates along intermediate and fast spreading mid-ocean ridges, Geophys. J. R. Astron. Soc., 87, 421-454, 1986.

Courtney, R. C., and R. S. White, Anomalous heat flow and geoid across the Cape Verde Rise: Evidence for dynamic support from a thermal plume in the mantle, Geophys. J. R. Astron. Soc., 87, $815-867,1986$.

Crough, S. T., Thermal origin of mid-plate hot-spot swells, Geophy. J. R. Astron. Soc., 55, 451-469, 1978.

Davis, E. E., Thermal aging of oceanic lithosphere, in Handbook of Seafloor Heat Flow, edited by J. A. Wright and K. E. Louden, pp. 145-167, CRC Press, Boca Raton, Fla., 1989.

Davis, E. E., and C. R. B. Lister, Heat flow measured over the Juan de Fuca Ridge: Evidence for widespread hydrothemal circulation in a highly heat transportive crust, J. Geophys. Res., 82, 4845-4860, 1977.

Davis, E. E., C. R. B. Lister, and J. G. Sclater, Towards determining the thermal state of old ocean lithosphere: Heat-how measurements from the Blake-Bahama outer ridge, north-westem Atlantic, Geophys. J. R. Astron. Soc, $78,507-545,1984$.

Detrick, R. S., and S. T. Crough, Island subsidence, hot spots, and lithosphere thinning, J. Geophys. Res., 83, 1236-1244, 1978.

Detrick, R. S., R. P. Von Herzen, B. Parsons, D. Sandwell, and M. Dougherty, Heat flow observations on the Bermuda Rise and thermal models of midplate swells, J. Geophys. Res., 91, 3701-3723, 1986.

Diament, D., and N. Baudry, Structural trends in the Southern Cook and Austral archipelagoes (south central Pacific) based on an analysis of Seasat data: Geodynamic implications, Earth Planet Sci. Lett., 85, 427-438, 1987.

Dunbar, J. A., and D. T. Sandwell, A boudinage model for crossgrain lineations, Eos Trans. AGU, 69, 1429, 1988.

Duncan, R. A., and D. A. Clague, Pacific plate motion recorded by linear volcanic chains, The Ocean Basins and Margins 7A: The 
Pacific Ocean, edited by A. E. Naim, F. G. Stehli, and S. Uyeda, pp. 89-121, Plenum, New York, 1985.

Halunen, A. J., and R. P. Von Herzen, Heat flow in the western equatorial Pacific Ocean, J. Geophys. Res., 78, 5195-5208, 1973.

Hart, S. R., A large-scale isotope anomaly in the southern hemisphere mantle, Nature, 309, 753-757, 1984.

Hart, S. R., Heterogeneous mantle domains: Signatures, genesis and mixing chronologies, Earth Planet. Sci. Lett., 90, 273-296, 1988.

Haxby, W. F., and J. K. Weissel, Evidence for small-scale mantle convection from Seasat altimeter data, J. Geophys. Res., 91, 3507-3520, 1986.

Herman, B. M., The thermal evolution of oceanic lithosphere in the South Atlantic, Ph.D. thesis, 347 pp., Columbia Univ., New York, 1987.

Hetenyi, M., Beams on Elastic Foundation, 255 pp., University of Michigan Press, Ann Arbor, 1974.

Kuzmin, V. A., A. E., Suzyumov, and A. V., Bezludov, Geothermic soundings on the Manihiki Plateau and the Marcus-Necker Rise (the Pacific Ocean), (in Russian), Okeanologiya, 12, 1044-1046, 1972.

Langseth, M. G., and P. T. Taylor, Recent heat flow measurements in the Indian Ocean, J. Geophys. Res., 72, 6249-6260, 1967.

Langseth, M. G., and R. P. Von Herzen, Heat flow through the floor of the world oceans, in The Sea, vol. 4, edited by A. E. Maxwell, pp. 299-352, Interscience, New York, 1970.

Langseth, M. G., P. J. Grim, and M. Ewing, Heat-flow measurements in the east Pacific Ocean, J. Geophys. Res., 70, 367-380, 1965.

Langseth, M. G., I. Malone, and D. Berger, Sea floor geothermal measurements from Vema cruise 24, 452 pp., Columbia Univ., Tech. Rept. 3-CU-3-71, New York, 1971. (Available as NTIS A0729682 from Natl. Tech. Inf. Serv., Springfield, Va.)

Lister, C. R. B., On the thermal balance of a mid-ocean ridge, Geophys. J. R. Astron. Soc., 26, 515-535, 1972.

Lister, C. R. B., J. G. Sclater, E. E. Davis, H. Villinger, and S. Nagihara, Heat flow maintained in oceanic basins of great age: Investigations in the north-equatorial west Pacific, Geophys. J. Int., 102 $603-630,1990$

Liu, M., and C. G. Chase, Evolution of midplate hotspot swells: Numerical solutions, J. Geophys. Res., 94, 5571-5584, 1989.

Louden, K. E., Marine heat flow data listung, Appendix B, in Handbook of Seafloor Heat Flow, pp. 325-485, edited by J. A. Wright and K. E. Louden, CRC Press, Boca Raton, Fla., 1989.

Louden, K. E., D. O. Wallace, and R. C. Courney, Heat flow and depth versus age for the Mesozoic northwest Atlantic Ocean: Results from the Sohm abyssal plain and implications for the Bermuda Rise, Earth Planet. Sci. Lett., 83, 109-122, 1987.

Ludwig, W. J. and R. E. Houtz, Isopach map of sediments in the Pacific Ocean basin and marginal sea basins, Am. Assoc. Pet. Geol., Tulsa, Okla., 1979.

Mammerickx, J., R. N. Anderson, H. W. Menard, and S. M. Smith, Morphology and tectonic evolution of the east-central Pacific, Geol. Soc. Am. Bull., 86, 111-118, 1975.

Marty, J. C., and A. Cazenave, Regional variations in subsidence rate of oceanic plates: A global analysis, Earth Planet. Sci. Let., 94, 301-315, 1989

Maxwell, A., The outflow of heat under the Pacific Ocean, Ph.D. thesis, Univ. of Calif., Los Angeles, 1958.

McAdoo, D. C., and D. T. Sandwell, On the source of cross-grained lineations in the central Pacific gravity field, J. Geophys. Res., 94, 9341-9352, 1989.

McNutt, M. K., Temperature beneath midplate swells: The inverse problem, in Seamounts, Islands, and Atolls, Geophys. Monogr. Ser., vol. 43, edited by B. H. Keating, P. Fryer, R. Batiza, and G. W. Boehlert, 123-132, AGU, Washington, D.C., 1987.

McNutt, M. K., and K. M. Fisher, The South Pacific superswell, in Seamounts, Islands, and Atolls, Geophys. Monogr. Ser., vol. 43, edited by B.H. Keating, P. Fryer, R. Batiza, and G. W. Boehlert, pp. 25-34, AGU, Washington, D.C., 1987.

McNutt, M. K., and A. V. Judge, The superswell and mantle dynamics beneath the South Pacific, Science, 248, 969-975, 1990.

McNutt, M., and H. W. Menard, Lithospheric flexure and uplifted atolls, J. Geophys. Res., 83, 1206-1212, 1978.

McNutt, M. K., K. M. Fisher, S. Kruse, and J. Natland, The origin of the Marquesas fracture zone ridge and its implications for the nature of hot spots, Earth Planet. Sci. Lett., 91, 381-393, 1989.

McNutt, M. K., E. L. Winterer, W. W. Sager, J. H. Natland, G. Ito, The Darwin Rise: A Cretaceous superswell?, Geophys. Res. Lett., 17, 1101-1104, 1990.
Menard, H. W., Marine Geology of the Pacific, 271 pp., McGraw-Hill, New York, 1964.

Menard, H. W., Darwin reprise, J. Geophys. Res., 89, 9960-9968, 1984.

Menard, H. W., and M. McNutt, Evidence for and consequences of thermal rejuvenation, J. Geophys. Res., 87, 8570-8580, 1982.

Morgan, W. J., Plate motions and deep mantle convection, Geol. Soc. Am. Bull., 132, 7-22, 1972.

Natland, J. H., and E. Wright, Magmatic lineages and mantle sources of Cretaceous seamount in the central Pacific, Eos Trans. AGU, 65, 1075-1076, 1984.

Nishimura, C. E., and D. W. Forsyth, Anomalous Love-wave phase velocities in the Pacific: Sequential pure-path and spherical harmonic inversion, Geophys. J. R. Astron. Soc, 81, 389-407, 1985.

Okal, E. A., and R. Batiza, Hotspots: The first 25 years, in Seamounts, Islands, and Atolls, Geophys. Monogr. Ser., vol. 43, edited by B.H. Keating, P. Fryer, R. Batiza, and G.W. Boehlert, pp. 1-11, AGU, Washington, D.C., 1987.

Okal, E. A., and A. Cazenave, A model for the plate tectonic evolution of the east-central Pacific based on Seasat investigations, Earth Planet. Sci. Lett., 72, 99-116, 1985.

Parsons, B., and S. Daly, The relationship between surface topography, gravity anomalies, and temperature structure of convection, $J$. Geophys. Res., 88, 1129-1144, 1983.

Parsons, B., and J. G. Sclater, An analysis of the variation of ocean floor bathymetry and heat flow with age, J. Geophys. Res., 82, 803-827, 1977.

Renkin, M. L., and J. G. Sclater, Depth and age in the North Pacific, J. Geophys. Res., 93, 2919-2935, 1988.

Robinson, E. M., and B. Parsons, Effect of a shallow low-viscosity zone on the formation of midplate swells, J. Geophys. Res., 93, 3144-3156, 1988.

Schlanger, S. O., H. C. Jenkyns, and I. Premoli-Silva, Volcanism and vertical tectonics in the Pacific basin related to global Cretaceous transgressions, Earth Planet. Sci. Lett., 52, 435-449, 1981.

Sclater, J. G. and J. Francheteau, The implications of terrestrial heat flow observations on current tectonic and geochemical models of the crust and upper mantle of the Earth, Geophys. J. R. Astron. Soc., 20 $509-542,1970$.

Sclater, J. G., U. G. Ritter, and F. S. Dixon, Heat flow in the southwestem Pacific, J. Geophys. Res., 77, 5697-5708, 1972.

Sclater, J. G., J. Crowe, and R. N. Anderson, On the reliability of oceanic heat flow averages, J. Geophys. Res., 81, 2997-3006, 1976.

Sclater, J. G., C. Jaupart, and D. Galson, The heat flow through oceanic and continental crust and the heat loss of the Earth, Rev. Geophys., $18,269-311,1980$.

Sleep, N. H., Hotspots and mantle plumes: Some phenomenology, $J$. Geophys. Res., 95, 6715-6736, 1990.

Smith, W. H. F., H. Staudigel, A. B. Watts, and M. S. Pringle, The Magellan Seamounts: Early Cretaceous record of the South Pacific isotopic and themal anomaly, $J$. Geophys. Res., 94, 10,501-10,523, 1989.

Staudigel, H., K. H. Park, M. Pringle, J. L. Rubenstone, W. S. F. Smith, and A. Zindler, The longevity of the South Pacific isotopic and thermal anomaly, Earth Planet. Sci. Lett., 102, 24-44, 1991.

Stein, C. A., and D. H. Abbott, Implications of estimated and measured thermal conductivity for oceanic heat flow studies, Mar. Geophys. Res., in press, 1991.

Stein, C. A., and J. K. Weissel, Constraints on the central Indian basin themnal structure from heat flow, seismicity and bathymetry, Tectonophysics., 176, 315-332, 1990.

Stein, C. A., S. Cloetingh, and R. Wortel, Seasat-derived gravity constraints on stress and deformation in the northeastem Indian Ocean. Geophys. Res. Lett., 16, 823-826, 1989.

Tracy, R. J., Petrology and genetic significance of an ultramafic xenolith suite from Tahiti, Earth Planet. Sci. Lett., 48, 80-96, 1980.

Turcotte, D. L., and G. Schuber, Geodynamics: Applications of Continuum Physics to Geological Problem, John Wiley, New York, 1982.

Turner, D. L., and R. D. Jarrard, K-Ar dating of the Cook-Austral island chain: A test of the hotspot hypothesis, J. Volcanol. Geotherm. Res., $12,187-220,1982$

Vacquier, V., S. Uyeda, M. Yasui, J. Sclater, C. Corry, and T. Watanabe, Studies of the thermal state of the Earth., The 19th paper: Heat-flow measurements in the northwestem Pacific, Bull. Earthquake Res. Inst., Univ. Tokyo, 44, 1519-1535, 1966.

Von Herzen, R., Heat-flow values from the southeastern Pacific, Nature, 183, 882-883, 1959.

Von Herzen, R., and S. Uyeda, Heat flow through the eastem Pacific Ocean floor, J. Geophys. Res., 68, 4219-4250, 1963. 
Von Herzen, R. P., R. S. Detrick, S. T. Crough, D. Epp and U. Fehn, Themal origin of the Hawaiian swell: Heat flow evidence and thermal models, J. Geophys. Res., 87, 6711-6723, 1982.

Von Herzen, R. P., J. J. Cordery, R. S. Detrick, and C. Fang, Heat flow and the thermal origin of the hot spot swells: The Hawaiian swell revisited, J. Geophys. Res., 94, 13,783-13,799, 1989.

Watts, A. B., J. H. Bodine and M. S. Steckler, Observations of flexure and the state of stress in the oceanic lithosphere, J. Geophys. Res., 85, 6369-6376, 1980 .

Williams, D. L., R. P. Von Herzen, J. G. Sclater, and R. N. Anderson, The Galapagos spreading centre: Lithospheric cooling and hydrothermal circulation, Geophys. J. R. Astron. Soc., 38, 587-608, 1974.

Wolfe, C. J. and M. K. McNutt, Compensation of Cretaceous seamounts of the Darwin Rise, Northwest Pacific Ocean, J. Geophys. Res., 96, 2363-2374, 1991.

Woods, M. T., J. J. Leveque, E. A. Okal, and M. Cara, Two-station measurements of Rayleigh wave group velocity along the Hawai'ian Swell, Geophys. Res. Lett, 18, 105-108, 1991.

Wysession, M. E., E. A. Okal, and K. L. Miller, Intraplate seismicity of the Pacific basin, 1913-1988, Pure Appl. Geophys., 135, 261-359. 1991.

D. H. Abboth, Lamont-Doherty Geological Observatory, Columbia University, Palisades, NY 10964.

C. A. Stein, Department of Geological Sciences, University of Illinois at Chicago, Box 4348, m/c 186. Chicago, IL 60680 .

Received July 16, 1990

revised March 5, 1991;

accepted March 11, 1991.) 